Homology, Homotopy and Applications, vol.5(1), 2003, pp.261-296

\title{
(CO)HOMOLOGY OF CROSSED MODULES WITH COEFFICIENTS IN A $\pi_{1}$-MODULE
}

\author{
SIMONA PAOLI \\ (communicated by Graham Ellis)
}

\begin{abstract}
We define a cotriple (co)homology of crossed modules with coefficients in a $\pi_{1}$-module. We prove its general properties, including the connection with the existing cotriple theories on crossed modules. We establish the relationship with the (co)homology of the classifying space of a crossed module and with the cohomology of groups with operators. An example and an application are given.
\end{abstract}

\section{Introduction}

In this paper we present some developments in the (co)homology of crossed modules. In the work of Carrasco, Cegarra and Grandjean [4] the authors proved that the category of crossed modules is tripleable over the category of sets, hence it is an algebraic category; then they used the resulting cotriple to construct a (co)homology theory of crossed modules in the spirit of the Barr and Beck theory [3].

Later Grandjean, Ladra and Pirashvili [13] have proved that there is an exact homology sequence

$$
\cdots \rightarrow H_{n+1} B(T, G, \mu) \rightarrow \zeta H_{n}^{C C G}(T, G, \mu) \rightarrow H_{n} G \rightarrow H_{n} B(T, G, \mu) \rightarrow \cdots
$$

which relates the integral homology of the classifying space of a crossed module and the cotriple homology of [4].

The (co)homology theory of [4] has trivial coefficients. In any algebraic category the passage from trivial coefficients for the (co)homology theory to global or local ones is achieved by a well known procedure which consists of taking abelian group objects in the slice category; the cohomology of a crossed module $\Phi$ with a system of global or local coefficients is equivalent to the cohomology in the slice category of $\mathrm{id}_{\Phi}$ with trivial coefficients. Although there is no theoretic difficulty in realizing this passage, to achieve it in practice in a concrete algebraic context like the one of crossed modules is not entirely trivial.

One of the first questions to consider is whether it is possible identify a manageable class of coefficients giving rise to a (co)homology theory of crossed modules which has interesting properties and leads to applications. This was one of our motivating questions which we have tried to answer in this paper by concentrating

Received June 14, 2002, revised July 17, 2003; published on July 28, 2003.

2000 Mathematics Subject Classification: 18G50 (18G30).

Key words and phrases: Crossed module, cotriple (co)homology, classifying space.

(C) 2003, Simona Paoli. Permission to copy for private use granted. 
our attention on two special cases of a system of local coefficients associated to a $\pi_{1}$-module, where $\pi_{1}$ is the first homotopy group of the crossed module.

Our motivation for considering $\pi_{1}$-module coefficients for the cotriple (co)homology comes from the long exact sequence (1). Since the (co)homology of the classifying space of a crossed module is defined in general with $\pi_{1}$-module coefficients (see [11]) it is natural to expect that an appropriate cotriple (co)homology of crossed modules with $\pi_{1}$-module coefficients would lead to long exact (co)homology sequences generalizing (1) in the homology case. One of our main results, Theorem 13, establishes precisely this.

Given a crossed module $\Phi$ with first homotopy group $\pi_{1}$, the canonical projection $\Phi \rightarrow\left(1, \pi_{1}, i\right) \equiv \pi_{1}(\Phi)$ induces a functor

$$
\left(\mathbf{C M} / \pi_{1}(\Phi)\right)_{a b} \rightarrow(\mathbf{C M} / \Phi)_{a b} .
$$

A system of local coefficients for the cohomology of a crossed module $\Phi$ could be defined as an object of $\left(\mathbf{C M} / \pi_{1}(\Phi)\right)_{a b}$. The functor (2) takes this system of local coefficients into a system of global coefficients used to compute the (co)homology.

In this paper we work with two special cases of the above system of local coefficients; these correspond to the split extensions of crossed modules

$$
(A, 1,0) \longmapsto\left(A, \pi_{1}, 0\right) \rightarrow\left(1, \pi_{1}, i\right)
$$

and

$$
(1, A, i) \longmapsto\left(1, A \rtimes \pi_{1}, i\right) \rightarrow\left(1, \pi_{1}, i\right) .
$$

In Sections 2 to 6 we consider the coefficients corresponding to the split extension (3), and study the corresponding (co)homology theory. After recalling some background in Section 1, in Section 2 we introduce the (co)homology. For this purpose, the notions of module and derivation in the sense of categories of interest [17] are used to define a derivation functor, and a dual Diff functor, from crossed modules to abelian groups. We point out that the notions of action, extensions and semidirect product of crossed modules were also worked out by Norrie [16], internally in the category of crossed modules. This has been used by Vieites and Casas [22] to give a different approach to derivations of crossed modules.

In Section 3 we study the case of aspherical crossed modules; these form a subcategory isomorphic to the category of surjective group homomorphisms. We prove that our (co)homology for the aspherical crossed module corresponding to the surjective group homomorphism $f: G \rightarrow G^{\prime}$ is isomorphic, up to a dimension shift, to the relative group (co)homology of the pair $\left(G^{\prime}, G\right)$ defined by Loday [14]. Other general properties of the (co)homology are proved in Section 4. In Section 5 we establish the relationship between our theory and the (co)homology of the classifying space, recovering the result of $[\mathbf{1 3}]$ in the case of homology with integral coefficients. This result is illustrated with an example in Section 6 where we obtain some information about the (co)homology of the crossed module corresponding to a $\mathbb{Z} G$-module $M$.

The coefficients corresponding to the split extension (4) are treated in Section 7 . We prove that the corresponding cotriple (co)homology coincides with the (co)homology of the classifying space of the crossed module, up to a dimension shift 
of 1 , and in dimensions $n>0$. An application to the cohomology of the classifying space follows.

In the last section we elucidate the relationship between the cohomology of crossed modules with $\pi_{1}$-module coefficients introduced in Section 2 and the cohomology of groups with operators studied in [6]. In order to study the relationship between the two theories we establish the preliminary result, which may be of independent interest, that when $(T, G, \mu)$ is a precrossed module the cohomology $H_{G}^{*}(T, A)$ of $[\mathbf{6}]$ can be described as cohomology of precrossed modules with a system of local coefficients.

\section{Acknowledgments}

This paper is based on part of my thesis [18]. I am very grateful to my supervisor Alan Robinson for his advice and encouragement and to the Engineering and Physical Sciences Research Council for financial support. I thank the Georgian Academy of Sciences for their hospitality while part of this work was carried out; in particular I am grateful to Hvedri Inassaridze for suggesting the initial direction of my work and to Tamar Datuashvili and Teimuraz Pirashvili for useful conversations. I would also like to thank the referee for some helpful comments and suggestions.

\section{Preliminaries}

\subsection{Crossed modules.}

Recall that a crossed module $\Phi=(T, G, \mu)$ consists of a group homomorphism $\mu: T \rightarrow G$ and of an action of $G$ on $T$ such that

$$
\mu\left({ }^{g} t\right)=g \mu(t) g^{-1}, \quad{ }^{\mu(t)} t^{\prime}=t t^{\prime} t^{-1}
$$

for each $t, t^{\prime} \in T, g \in G$. A homomorphism of crossed modules $\left(f_{T}, f_{G}\right):(T, G, \mu) \rightarrow$ $\left(T^{\prime}, G^{\prime}, \mu^{\prime}\right)$ is a pair of group homomorphisms $f_{T}: T \rightarrow T^{\prime}, f_{G}: G \rightarrow G^{\prime}$ such that $\mu^{\prime} f_{T}=f_{G} \mu$ and $f_{T}\left({ }^{g} t\right)={ }^{f_{G}(g)} f_{T}(t)$ for all $g \in G, t \in T$. We denote by CM the category of crossed modules. This category has several equivalent descriptions.

Recall that a cat ${ }^{1}$-group consists of a group $G$ with two endomorphisms $d_{0}, d_{1}$ : $G \rightarrow G$ such that

$$
d_{1} d_{0}=d_{0}, \quad d_{0} d_{1}=d_{1}, \quad\left[\operatorname{ker} d_{0}, \operatorname{ker} d_{1}\right]=1 .
$$

A morphism of cat ${ }^{1}$-groups $\left(G, d_{0}, d_{1}\right) \rightarrow\left(G^{\prime}, d_{0}^{\prime}, d_{1}^{\prime}\right)$ is a group homomorphism $f: G \rightarrow G^{\prime}$ such that $d_{i}^{\prime} f=f d_{i} i=0,1$.

The category of crossed modules is equivalent to the category of cat ${ }^{1}$-groups [15]. Given a crossed module $(T, G, \mu)$, the corresponding cat ${ }^{1}$-group is $\left(T \rtimes G, d_{0}, d_{1}\right)$, $d_{0}(t, g)=(1, g), d_{1}(t, g)=(1, \mu(t) g)$ for all $(t, g) \in T \rtimes G$.

Another description of the category of crossed modules is given by its equivalence with the category $\mathbf{S G}_{\leqslant 1}$ of simplicial groups whose Moore complex has length $1[\mathbf{1 5}]$. An object of $\mathbf{S G}_{\leqslant 1}$ is a simplicial group $G_{*}$ such that $N_{i}\left(G_{*}\right)=0$ for $i>1$ while $N_{1}\left(G_{*}\right) \neq 0$ where $N_{*}: \mathbf{S G}_{\leqslant 1} \rightarrow \mathbf{C M}$ is the Moore normalization functor. This is 
defined by

$$
N_{n} G_{*}=\underset{i>0}{\cap} \operatorname{ker}\left(\partial_{i}^{n}\right) \quad n>0
$$

with boundary map $d: N_{n} G_{*} \rightarrow N_{n-1} G_{*}, d=\partial_{\left.0\right|_{N_{n} G_{*}}}$. There is a functor $N_{*}^{-1}$ : $\mathbf{C M} \rightarrow \mathbf{S G}_{\leqslant 1}$ with $N_{*}^{-1}\left(N_{*} G\right) \cong G_{*}$ which is given by

$$
\begin{array}{ll}
N_{n}^{-1}(T, G, \mu)=T^{n} \rtimes G & n \geqslant 0, \\
\partial_{i}\left(t_{1}, \ldots, t_{n}, g\right)=\left(t_{1}, \ldots, \hat{t}_{i}, \ldots, t_{n}, g\right) & 1 \leqslant i \leqslant n, \\
\partial_{0}\left(t_{1}, \ldots, t_{n}, g\right)=\left(t_{2} t_{1}^{-1}, \ldots, t_{n} t_{1}^{-1}, \mu t_{1} g\right) & \\
s_{i}\left(t_{1}, \ldots, t_{n}, g\right)=\left(t_{1}, \ldots, t_{i}, 0, \ldots, t_{n}, g\right) . &
\end{array}
$$

Crossed modules are algebraic models for connected spaces which have trivial homotopy groups in dimension $n>2$, called 2-types (see for instance [15]). To any crossed module $(T, G, \mu)$ one can associate a connected $\mathrm{CW}$-space $B(T, G, \mu)$ called its classifying space with

$$
\pi_{1} B(T, G, \mu) \cong G / \mu(T), \quad \pi_{2} B(T, G, \mu) \cong \operatorname{ker} \mu, \quad \pi_{n} B(T, G, \mu) \cong 0 \text { for } n>2 .
$$

$B(T, G, \mu)$ is defined as the classifying space of the simplicial group $N_{*}^{-1}(T, G, \mu)$.

The homotopy groups of a crossed module $(T, G, \mu)$ are defined as $\pi_{1}=G / \mu(T)$, $\pi_{2}=\operatorname{ker} \mu, \pi_{n}=0$ for $n \neq 1,2$. A morphism of crossed modules is called a weak equivalence if it induces isomorphisms of homotopy groups.

It can be proved (see for instance [15]) that the functor $B(-)$ induces an equivalence between the homotopy category of connected 2-types and the localization of the category of crossed modules with respect to weak equivalences.

\subsection{CCG (co)homology.}

In [4] it is proved that the category of crossed modules is tripleable over Set, hence it is an algebraic category. It is shown there that the functor $\mathcal{U}: \mathbf{C M} \rightarrow$ Set, $\mathcal{U}(T, G, \mu)=T \times G$ has a left adjoint $\mathcal{F}:$ Set $\rightarrow \mathbf{C M}$. This is given by

$$
\mathcal{F}(X)=(\overline{F(X)}, F(X) * F(X), i)
$$

where $F(X)$ is the free group on $X, *$ is the free product, $i$ is the inclusion, $\overline{F(X)}$ is the kernel of the map $p_{2}: F(X) * F(X) \rightarrow F(X)$ determined by $p_{2} u_{1}=0$, $p_{2} u_{2}=\mathrm{id}, u_{1}, u_{2}$ being the coproduct injections.

It is proved in [4] that $\mathcal{U}$ is tripleable. This identifies the regular epimorphisms in $\mathbf{C M}$ as those homomorphisms $\left(f_{T}, f_{G}\right):(T, G, \mu) \rightarrow\left(T^{\prime}, G^{\prime}, \mu^{\prime}\right)$ such that $f_{T}$ and $f_{G}$ are surjective. Hence for each set $X$ the crossed module $\mathcal{F}(X)$ is a projective object in $\mathbf{C M}$; this category has enough projectives since any crossed module $(T, G, \mu)$ admits the projective presentation $\mathcal{F} \mathcal{U}(T, G, \mu) \rightarrow(T, G, \mu)$.

Let $\mathbb{G}=\mathcal{F U}$ be the cotriple arising from the pair of adjoint functors $(\mathcal{F}, \mathcal{U})$. This cotriple is used in [4] to define a (co)homology theory of crossed modules as follows. Recall that given a crossed module $(T, G, \mu)$ its abelianisation is the abelian crossed module $(T, G, \mu)_{a b}=(T /[G, T], G /[G, G], \bar{\mu})$. For each $n \geqslant 1$ the $n^{\text {th }} C C G$ homology of $(T, G, \mu)$ is the crossed module

$$
H_{n}^{C C G}(T, G, \mu)=H_{n-1}\left(\mathbb{G}_{\bullet}(T, G, \mu)_{a b}\right) .
$$


If $(A, B, \delta)$ is an abelian crossed module (that is $A$ and $B$ are abelian groups and $B$ acts trivially on $A$ ), for each $n \geqslant 1$ the $n^{\text {th }} C C G$ cohomology of $(T, G, \mu)$ with coefficients in $(A, B, \delta)$ is the abelian group

$$
\begin{aligned}
& H_{C C G}^{n}((T, G, \mu),(A, B, \delta))=H^{n-1} \operatorname{Hom}_{\mathbf{C M}}\left(\mathbb{G}_{\bullet}(T, G, \mu),(A, B, \delta)\right) \cong \\
& \cong H^{n-1} \operatorname{Hom}_{\mathbf{A b ~ C M}}\left(\mathbb{G}_{\bullet}(T, G, \mu)_{a b},(A, B, \delta)\right) .
\end{aligned}
$$

\subsection{Crossed modules as category of interest.}

It is a known fact that the category of cat $^{1}$-groups is a category of groups with operations in the sense of [19]. Recall that this consists of the following data: a category of groups with a set of operations $\Omega=\Omega_{0} \cup \Omega_{1} \cup \Omega_{2}$ where $\Omega_{i}$ is the set of $i$-ary operations in $\Omega$ such that the group operations of identity, inverse and multiplication (denoted $0,-,+$ ) are elements of $\Omega_{0}, \Omega_{1}, \Omega_{2}$ respectively; one has $\Omega_{0}=\{0\}$ and certain compatibility conditions hold (see [19]); finally there is a set of identities $E$ which includes the group laws.

In the case of cat ${ }^{1}$-groups, $\Omega_{0}=\{0\}, \Omega_{1}=\{-\} \cup\left\{d_{0}, d_{1}\right\}, \quad \Omega_{2}=\{+\}$ and $E$ consists of the group laws and of the identities (5). The compatibility conditions in this case are that $d_{0}, d_{1}$ commute with + , hence they are group homomorphisms.

In the category of cat ${ }^{1}$-groups we therefore have the notions of singular object, module, semidirect product, derivation. For a discussion of these notions in any category of groups with operations see for instance [7] and [19].

A cat ${ }^{1}$-group $\left(A, d_{0}, d_{1}\right)$ is a singular object if $A$ is an abelian group. The corresponding crossed module is then an abelian crossed module. Given a cat ${ }^{1}$-group $\left(G, s_{0}, s_{1}\right),\left(A, d_{0}, d_{1}\right)$ is a $\left(G, s_{0}, s_{1}\right)$-module if $\left(A, d_{0}, d_{1}\right)$ is singular and there is a split extension of cat $^{1}$-groups

$$
\left(A, d_{0}, d_{1}\right) \longmapsto\left(Q, s_{0}^{\prime}, s_{1}^{\prime}\right) \leftrightarrows\left(G, s_{0}, s_{1}\right) .
$$

This split singular extension induces an action of $\left(G, s_{0}, s_{1}\right)$ on $\left(A, d_{0}, d_{1}\right)$; a derivation $D$ from $\left(G, s_{0}, s_{1}\right)$ into $\left(A, d_{0}, d_{1}\right)$ is a group derivation from $G$ into $A$ which commutes with the 1 -ary operations $\omega \in \Omega_{1} \backslash\{-\}$, that is such that $D s_{i}=d_{i} D$ for $i=0,1$.

Since $\mathbf{C M}$ is equivalent to the category of cat $^{1}$-groups, it can be considered itself as a category of groups with operations. Moreover, since $\mathbf{C M}$ is tripleable over Set and the set $\Omega_{2}$ of 2-ary operations just consists of group multiplication, it is in fact a category of interest in the sense of [17]. In this paper we shall use the interpretation in terms of extensions of the first and second cotriple cohomology in a category of interest given in $[\mathbf{2 1}]$.

\subsection{Crossed modules in the category of cat $^{1}$-groups.}

Let $\mathcal{C}^{1} \mathcal{G}$ denote the category of cat ${ }^{1}$-groups and $\mathcal{C}^{2} \mathcal{G}$ the category of cat ${ }^{2}$-groups; we refer to [15] for the definition of cat ${ }^{2}$-group. Since $\mathcal{C}^{1} \mathcal{G}$ is a category of groups with operators, from [19] the category $\mathbf{C M}\left(\mathcal{C}^{1} \mathcal{G}\right)$ of crossed modules in $\mathcal{C}^{1} \mathcal{G}$ is equivalent to the category $\operatorname{Cat}\left(\mathcal{C}^{1} \mathcal{G}\right)$ of internal categories in $\mathcal{C}^{1} \mathcal{G}$. On the other hand there is an equivalence of categories between $\operatorname{Cat}\left(\mathcal{C}^{1} \mathcal{G}\right)$ and $\mathcal{C}^{2} \mathcal{G}$, as explained for instance in the proof of $\left[\mathbf{1 0}, \mathrm{I}-6\right.$, Proposition 1.2.3]. It follows that $\mathbf{C M}\left(\mathcal{C}^{1} \mathcal{G}\right)$ is equivalent to $\mathcal{C}^{2} \mathcal{G}$. The correspondences giving this equivalence of categories can 
be easily made explicit from $[\mathbf{1 9}]$ and $[\mathbf{1 0}]$. The category $\mathcal{C}^{2} \mathcal{G}$ of cat $^{2}$-groups is also equivalent to the category $\mathrm{Crs}^{2}$ of crossed squares, [15]. Hence there is an equivalence of categories between $\operatorname{CM}\left(\mathcal{C}^{1} \mathcal{G}\right)$ and $C r s^{2}$. The correspondences giving this equivalence can be described explicitly as follows:

\section{Lemma 1.}

a) Let $\left(\left(H, d_{0}, d_{1}\right),\left(H^{\prime}, d_{0}^{\prime}, d_{1}^{\prime}\right), \alpha\right)$ be an object of $\mathbf{C M}\left(\mathcal{C}^{1} \mathcal{G}\right)$. Let $(T, G, \mu)$ and $\left(T^{\prime}, G^{\prime}, \mu^{\prime}\right)$ be the crossed modules corresponding to $\left(H, d_{0}, d_{1}\right)$ and $\left(H^{\prime}, d_{0}^{\prime}, d_{1}^{\prime}\right)$ respectively. Then the following is a crossed square

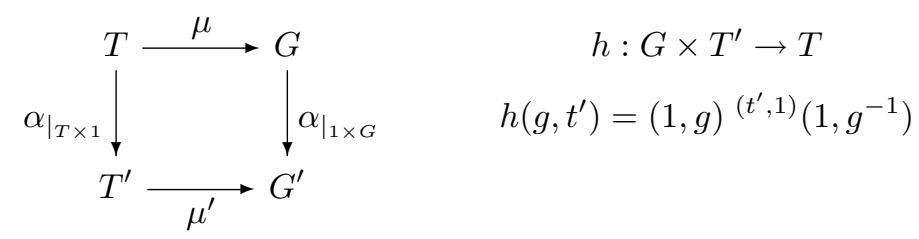

where $^{\left(t^{\prime}, 1\right)}\left(1, g^{-1}\right)$ is the crossed module action of $H^{\prime} \cong T^{\prime} \rtimes G^{\prime}$ on $H \cong T \rtimes G$.

b) Conversely, if

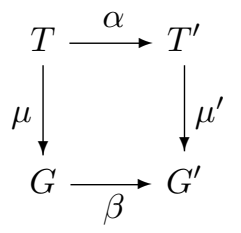

$h: T^{\prime} \times G \rightarrow T$

is a crossed square, the corresponding object of $\mathbf{C M}\left(\mathcal{C}^{1} \mathcal{G}\right)$ is

$$
\left(T \rtimes G, d_{0}, d_{1}\right) \stackrel{(\alpha, \beta)}{\longrightarrow}\left(T^{\prime} \rtimes G^{\prime}, d_{0}^{\prime}, d_{1}^{\prime}\right)
$$

where $d_{0}(t, g)=(1, g), \quad d_{1}(t, g)=(1, \mu(t) g), \quad d_{0}^{\prime}\left(t^{\prime}, g^{\prime}\right)=\left(1, g^{\prime}\right), \quad d_{1}^{\prime}\left(t^{\prime}, g^{\prime}\right)=$ $\left(1, \mu\left(t^{\prime}\right) g^{\prime}\right)$ for all $(t, g) \in T \rtimes G, \quad\left(t^{\prime}, g^{\prime}\right) \in T^{\prime} \rtimes G^{\prime}$ and the action of $T^{\prime} \rtimes G^{\prime}$ on $T \rtimes G$ is given by

$$
\left.\left(t^{\prime}, g^{\prime}\right)(t, g)=\left({t^{\prime}}^{g^{\prime}} t\right) h\left(t^{\prime}, g^{\prime} g\right), g^{g^{\prime}} g\right)
$$

for all $\left(t^{\prime}, g^{\prime}\right) \in T^{\prime} \rtimes G^{\prime}, \quad(t, g) \in T \rtimes G$.

Proof. It follows from the correspondences giving the equivalence of categories between $\mathbf{C M}\left(\mathcal{C}^{1} \mathcal{G}\right)$ and $\mathcal{C}^{2} \mathcal{G}$ (see [19] and [10]) and between $\mathcal{C}^{2} \mathcal{G}$ and $\operatorname{Crs}^{2}$ (see $[\mathbf{1 5}])$.

\section{Definition of the (co)homology and elementary properties}

In order to define our (co)homology theory, we first introduce Der and Diff functors on the category of crossed modules. We can do so in two equivalent ways, working directly in the category $\mathbf{C M}$ or working in the equivalent category of cat ${ }^{1}$ groups. We shall illustrate both ways in some detail. While the first one may be slightly more transparent, the reason we write explicitly the derivation functor in cat $^{1}$-groups is that viewing our (co)homology theory as (co)homology of cat ${ }^{1}$-groups 
will allow us to apply in the next sections the interpretation in terms of extensions of the first and second cotriple cohomology in a category of interest [21].

Let $\Phi=(T, G, \mu)$ be a crossed module, $A$ an abelian group. The abelian crossed module $(A, 1,0)$ is a $\Phi$-module if and only if there is a split extension of crossed modules

$$
(A, 1,0) \longmapsto\left(T^{\prime}, G^{\prime}, \mu^{\prime}\right) \leftrightarrows(T, G, \mu)
$$

Since the base group of the crossed module on the left is 1 the morphism $\left(T^{\prime}, G^{\prime}, \mu^{\prime}\right) \rightarrow$ $(T, G, \mu)$ is an isomorphism at the level of base groups and so we can assume $G^{\prime}=G$. Also observe that the section in (6) gives, by conjugation on $T^{\prime}$, an action of $T$ on $A$, hence $T^{\prime} \cong A \rtimes T$. So we can assume that the sequence (6) has the form

$$
(A, 1,0) \longmapsto\left(A \rtimes T, G, \mu^{\prime}\right) \leftrightarrows(T, G, \mu)
$$

where the morphisms on the left and on the right are the canonical inclusion and projection respectively. The action of $G$ on $A \rtimes T$ induces an action of $G$ on $A$; in fact, since $\left(\operatorname{pr}_{T}, \mathrm{id}_{G}\right)$ is a map of crossed modules, $\operatorname{pr}_{T}\left({ }^{g}(a, 1)\right)=1$ for all $g \in G, a \in A$. Also, since the splitting $\left(i_{T}, \operatorname{id}_{G}\right)$ is a map of crossed modules, $\left(0,{ }^{g} t\right)={ }^{g}(0, t)$ for all $g \in G, \quad t \in T$. It follows that, for all $g \in G, \quad t \in T, \widetilde{\mu}(a, t)=\mu(t)$, ${ }^{g}(a, t)={ }^{g}(a, 1){ }^{g}(0, t)=\left({ }^{g} a, 1\right)\left(0,{ }^{g} t\right)$ and $\widetilde{\mu}(a, t)=\mu(t)$. Requiring that the Peiffer identity holds for the crossed module $(A \rtimes T, G, \widetilde{\mu})$ an easy calculation shows that for each $a, a^{\prime} \in A, \quad t, t^{\prime} \in T$

$$
{ }^{\mu(t)} a^{\prime}=a+{ }^{t} a^{\prime}-{ }^{t t^{\prime}} t^{-1} a .
$$

It follows that $a={ }^{t} a={ }^{\mu(t)} a$ for each $a \in A, \quad t \in T$. Hence $A$ is a $\pi_{1}$-module, where $\pi_{1}=G / \mu(T)$. We denote $A \rtimes \Phi=(A \times T, G, \widetilde{\mu})$. From a general fact in algebraic categories,

$$
\operatorname{Der}(\Phi,(A, 1,0)) \cong \operatorname{Hom}_{\mathbf{C M} / \Phi}(\Phi, A \rtimes \Phi) .
$$

Since $A \rtimes \Phi=(A \times T, G, \widetilde{\mu})$ it is straightforward that $\operatorname{Hom}_{\mathrm{CM} / \Phi}(\Phi, A \rtimes \Phi) \cong$ $\operatorname{Hom}_{G}(T, A)$, where $\operatorname{Hom}_{G}(T, A)$ is the group of $G$-equivariant homomorphisms from $T$ to $A$. In conclusion we obtain

$$
\operatorname{Der}((T, G, \mu),(A, 1,0)) \cong \operatorname{Hom}_{G}(T, A) .
$$

The same procedure can be repeated in the equivalent category of cat $^{1}$-groups; let $(T \rtimes G, d, s), \quad(A, 0,0)$ be the cat $^{1}$-groups corresponding to the crossed modules $(T, G, \mu)$ and $(\mathrm{A}, 1,0)$ respectively. It is easy to see that $(A, 0,0)$ is a $(T \rtimes G, d, s)$ module if and only if there is a split extension of cat ${ }^{1}$-groups

$$
(A, 0,0) \longmapsto\left(A \rtimes(T \rtimes G), d^{\prime}, s^{\prime}\right) \leftrightarrows(T \rtimes G, d, s)
$$

where $d^{\prime}(a,(t, g))=(0, d(t, g)), \quad s^{\prime}(a,(t, g))=(0, s(t, g)), \quad a \in A, \quad(t, g) \in T \rtimes G$. Requiring that the identity $\left[\operatorname{ker} d^{\prime}, \operatorname{ker} s^{\prime}\right]=1$ holds, an easy calculation shows that, for each $a, a^{\prime} \in A, t, t^{\prime} \in T$

$$
a+{ }^{(t, 1)} a^{\prime}-{ }^{\left(t^{\prime}, \mu\left(t^{\prime}\right)^{-1}\right)} a-a^{\prime}=0 .
$$

It follows that $a={ }^{(t, 1)} a={ }^{(1, \mu(t))} a$ for each $a \in A, t \in T$. Thus $T \rtimes G$ acts on $A$, $T \times 1$ and $1 \times \mu(T)$ act trivially on $A$, so $A$ is a $\pi_{1}$-module, $\pi_{1}=G / \mu(T)$. 
From [17] a derivation from $(T \rtimes G, d, s)$ into $(A, 0,0)$ is a map $D: T \rtimes G \rightarrow A$ which is a group derivation and such that it commutes with the 1-ary operations $\omega \in \Omega_{1} \backslash\{-\}$. Hence $D(d(t, g))=D(s(t, g))=0$ for $(t, g) \in T \rtimes G$, so that $D(1, g)=$ 0 for every $g \in G$.

In conclusion

$$
\operatorname{Der}((T \rtimes G, d, s),(A, 0,0)) \cong\{D \in \operatorname{Der}(T \rtimes G, A) \mid D(1, G)=0\} .
$$

The two approaches are clearly equivalent. In fact there is an isomorphism

$$
\alpha:\{D \in \operatorname{Der}(T \rtimes G, A) \mid D(1, G)=0\} \rightarrow \operatorname{Hom}_{G}(T, A)
$$

given by $\alpha(D)(t)=D(t, 1)$, as easily checked. This motivates the following definition.

Definition 2. Let $\Phi=(T, G, \mu)$ be a crossed module, $A$ an abelian group, $\pi_{1}=$ $G / \mu(T)$. We say that $\Phi$ acts on $A$ if $A$ is a $\pi_{1}$-module. In this case we define

$$
\operatorname{Der}(\Phi, A)=\{D \in \operatorname{Der}(T \rtimes G, A) \mid D(1, G)=0\} \cong \operatorname{Hom}_{G}(T, A) .
$$

where $\operatorname{Der}(T \rtimes G, A)$ denotes group derivations from $T \rtimes G$ into $A$ and the action of $T \rtimes G$ on $A$ is given by $(t, g) a={ }^{g \mu(T)} a$.

Similarly if $\Phi$ acts on $A$ we have a contravariant functor $\operatorname{Der}(-, A): \mathbf{C M} / \Phi \rightarrow \mathbf{A b}$ on the slice category; in fact, given an object $\Phi^{\prime} \rightarrow \Phi$ of $\mathbf{C M} / \Phi$, the action of $\Phi$ on $A$ induces an action of $\Phi^{\prime}$ on $A$. When the context is clear, given an action of $\Phi$ on $A$ we will write $\operatorname{Der}\left(\Phi^{\prime} \rightarrow \Phi, A\right)$ as $\operatorname{Der}\left(\Phi^{\prime}, A\right)$.

Given a crossed module $\Phi=(T, G, \mu)$ let $J_{T, G}$ be the ideal of $\mathbb{Z}(T \rtimes G)$ generated by $\{(1, g)-(1,1) \mid 1 \neq g \in G\}$. Then

$$
\operatorname{Der}(\Phi, A) \cong \operatorname{Hom}_{\mathbb{Z}(T \rtimes G)}\left(\frac{\mathfrak{I}_{\mathbb{Z}(T \rtimes G)}}{J_{T, G}}, A\right) \cong \operatorname{Hom}_{\mathbb{Z} \pi_{1}}\left(\mathbb{Z} \pi_{1} \otimes_{\mathbb{Z}(T \rtimes G)} \frac{\mathfrak{I}_{\mathbb{Z}(T \rtimes G)}}{J_{T, G}}, A\right) .
$$

This motivates our next definition.

Definition 3. Let $\Phi=(T, G, \mu)$ be a crossed module. Define

$$
\text { Diff } \Phi=\mathbb{Z} \pi_{1} \otimes_{\mathbb{Z}(T \rtimes G)} \frac{\mathfrak{I}_{\mathbb{Z}(T \rtimes G)}}{J_{T, G}} .
$$

For a crossed module $\Phi$ acting on the abelian group $A$, we denote $\operatorname{Diff}(\Phi, A)=$ $A \otimes_{\mathbb{Z} \pi_{1}} \operatorname{Diff} \Phi$. Similarly we have a covariant functor Diff $(-, A): \mathbf{C M} / \Phi \rightarrow \mathbf{A b}$.

The slice category $\mathbf{C M} / \Phi$ is tripleable over $\operatorname{Set} / \mathcal{U}(\Phi)$ and we shall denote by $\mathbb{G}$ the corresponding cotriple. We now consider the cotriple (co)homology of $\Phi$ with coefficients in the $\Phi$-module $(A, 1,0)$. This is the left (resp. right) derived functor of the functor Diff $(-, A)$ (resp. Der $(-, A)$ ) on the slice category $\mathbf{C M} / \Phi$ with respect to the cotriple $\mathbb{G}$.

Definition 4. Let $\Phi=(T, G, \mu)$ be a crossed module, $A$ a $\pi_{1}$-module. Define for each $n \geqslant 0$

$$
\begin{aligned}
& D^{n}(\Phi, A)=H^{n} \operatorname{Der}\left(\mathbb{G}_{\bullet} \Phi, A\right) \\
& D_{n}(\Phi, A)=H_{n} \operatorname{Diff}\left(\mathbb{G}_{\bullet} \Phi, A\right) .
\end{aligned}
$$


The following are elementary properties of the (co)homology which can be deduced from well known general facts about cotriple (co)homology in an algebraic category $[\mathbf{3}]$. In what follows a projective crossed module means a projective object in the category of crossed modules.

Proposition 5. Let $\Phi=(T, G, \mu)$ be a crossed module acting on the abelian group A. Then

a) $D^{0}(\Phi, A) \cong \operatorname{Der}(\Phi, A), \quad D_{0}(\Phi, A) \cong \operatorname{Diff}(\Phi, A)$.

b) If $\Phi$ is a projective crossed module, $D^{n}(\Phi, A)=0, \quad D_{n}(\Phi, A)=0$ for each $n>0$.

c) Any short exact sequence of $\pi_{1}$-modules $0 \rightarrow A \rightarrow A^{\prime} \rightarrow A^{\prime \prime} \rightarrow 0$ induces long exact (co)homology sequences

$\cdots \rightarrow D^{n}(\Phi, A) \rightarrow D^{n}\left(\Phi, A^{\prime}\right) \rightarrow D^{n}\left(\Phi, A^{\prime \prime}\right) \rightarrow D^{n+1}(\Phi, A) \rightarrow \cdots$

$\cdots \rightarrow D_{n}(\Phi, A) \rightarrow D_{n}\left(\Phi, A^{\prime}\right) \rightarrow D_{n}\left(\Phi, A^{\prime \prime}\right) \rightarrow D_{n-1}(\Phi, A) \rightarrow \cdots$

\section{3. (Co)homology of aspherical crossed modules}

A crossed module $\Phi=(T, G, \mu)$ is called aspherical when the map $\mu$ is injective. The category of aspherical crossed modules is isomorphic to the category of surjective group homomorphisms. Given a surjective group homomorphism $f: G \rightarrow G^{\prime}$ the corresponding aspherical crossed module is $(\operatorname{ker} f, G, i)$ and will be denoted by $\Phi_{f}$. If $\Phi_{f}$ acts on the abelian group $A$, then $A$ is a $\mathbb{Z} G^{\prime}$-module as well as a $\mathbb{Z} G$-module via $f$. We say in this case that $A$ is an $f$-module and we denote $\operatorname{Der}\left(\Phi_{f}, A\right)=\operatorname{Der}(f, A), \operatorname{Diff} \Phi_{f}=\operatorname{Diff} f$.

Lemma 6. Let $f: G \rightarrow G^{\prime}$ be a surjective group homomorphism, $N=\operatorname{ker} f, A$ an $f$-module. Then

a) $\operatorname{Der}(f, A) \cong \operatorname{Hom}_{\mathbb{Z} G^{\prime}}\left(N_{a b}, A\right), \quad \operatorname{Diff} f \cong N_{a b}$.

b) Suppose that there is a group homomorphism $f^{\prime}: G^{\prime} \rightarrow G$ with $f f^{\prime}=$ id. Then there are short exact sequences

$$
\begin{aligned}
& 0 \rightarrow \operatorname{Der}\left(G^{\prime}, A\right) \rightarrow \operatorname{Der}(G, A) \rightarrow \operatorname{Der}(f, A) \rightarrow 0 \\
& 0 \rightarrow A \otimes_{\mathbb{Z} G^{\prime}} \text { Diff } f \rightarrow A \otimes_{\mathbb{Z} G} \Im_{G} \rightarrow A \otimes_{\mathbb{Z} G^{\prime}} \Im_{G^{\prime}} \rightarrow 0 .
\end{aligned}
$$

\section{Proof.}

a) Let $\alpha: \operatorname{Der}(f, A) \rightarrow \operatorname{Hom}_{\mathbb{Z} G^{\prime}}\left(N_{a b}, A\right)$ be defined by

$$
\alpha(D)(n[N, N])=D(n, 1), \quad n \in N .
$$

It is straightforward that $\alpha(D)$ is well defined; it is also a $\mathbb{Z} G^{\prime}$-homomorphism since, for each $g^{\prime}=f(g) \in G^{\prime}, n \in N$

$$
\begin{aligned}
& \alpha(D)\left(g^{\prime} \cdot n[N, N]\right)=\alpha(D)\left(g n g^{-1}[N, N]\right)=D\left((1, g)(n, 1)\left(1, g^{-1}\right)\right)= \\
& =(1, g) D(n, 1)=g^{\prime} \alpha(D)(n[N, N]) .
\end{aligned}
$$

Let $\beta: \operatorname{Hom}_{\mathbb{Z} G^{\prime}}\left(N_{a b}, A\right) \rightarrow \operatorname{Der}(f, A)$ be defined by

$$
\beta(\varphi)(n, g)=\varphi(n[N, N]), \quad(n, g) \in N \rtimes G .
$$


Then $\beta(\varphi)$ is a derivation and $\beta(\varphi)(1, G)=0$. We have $\alpha \beta(\varphi)(n[N, N])=$ $\beta(\varphi)(n, 1)=\varphi(n[N, N])$ for each $n \in N$; for each $D \in \operatorname{Der}(f, A), \quad(n, g) \in N \rtimes G$ it is $\beta \alpha(D)(n, g)=\alpha(D)(n[N, N])=D(n, 1)=D(n, g)$. Thus $\alpha$ and $\beta$ are inverse bijections and $\operatorname{Der}(f, A) \cong \operatorname{Hom}_{\mathbb{Z} G^{\prime}}\left(N_{a b}, A\right)$. Since this isomorphism holds for each $\mathbb{Z} G^{\prime}$-module $A$, Yoneda Lemma implies Diff $f \cong N_{a b}$.

b) Let $\alpha: \operatorname{Der}(G, A) \rightarrow \operatorname{Der}(f, A)$ be defined by $\alpha(D)(n, g)=D(n), D \in \operatorname{Der}(G, A)$, $(n, g) \in N \rtimes G$. If $\xi \in \operatorname{Der}(f, A)$, let $D(g)=\xi\left(g f^{\prime} f\left(g^{-1}\right), 1\right), g \in G$. For each $g_{1}, g_{2} \in G$

$$
\begin{aligned}
& \xi\left(g_{1} g_{2} f^{\prime} f\left(g_{2}^{-1}\right) f^{\prime} f\left(g_{1}^{-1}\right), 1\right)=\xi\left(\left(1, g_{1}\right)\left(g_{2} f^{\prime} f\left(g_{2}^{-1}\right), 1\right)\left(1, g_{1}^{-1}\right)\left(g_{1} f^{\prime} f\left(g_{1}^{-1}\right), 1\right)=\right. \\
& =\xi\left(g_{1} f^{\prime} f\left(g_{1}^{-1}\right), 1\right)+\left(1, g_{1}\right) \xi\left(g_{2} f^{\prime} f\left(g_{2}^{-1}\right), 1\right) .
\end{aligned}
$$

This shows that $D$ is a derivation. Also, $(\alpha D)(n, g)=D(n)=\xi(n, 1)=\xi(n, g)$ so $\alpha$ is surjective. Exactness at the other terms is straightforward and b) follows for the cohomology case.

From the well known exact sequence $N_{a b} \longmapsto \mathbb{Z} G^{\prime} \otimes_{\mathbb{Z} G} \mathfrak{I}_{G} \rightarrow \mathfrak{I}_{G^{\prime}}$ and from part a) we obtain the exact sequence

$$
A \otimes_{\mathbb{Z} G^{\prime}} \text { Diff } f \stackrel{\beta^{\prime}}{\rightarrow} A \otimes_{\mathbb{Z} G} \Im_{G} \stackrel{\alpha^{\prime}}{\rightarrow} A \otimes_{\mathbb{Z} G^{\prime}} \Im_{G^{\prime}} \rightarrow 0
$$

where $\beta^{\prime}(a \otimes n[N, N])=a \otimes(n-1), \quad a \in A, n \in N, \quad \alpha^{\prime}\left(a \otimes \sum_{i} b_{i}\left(g_{i}-1\right)\right)=$ $a \otimes \sum_{i} b_{i}\left(f\left(g_{i}\right)-1\right), \quad a \in A, b_{i} \in \mathbb{Z}, \quad 1 \neq g_{i} \in G$. Let $\gamma: A \otimes_{\mathbb{Z} G} \Im_{G} \rightarrow A \otimes_{\mathbb{Z} G^{\prime}} \operatorname{Diff} f$ be defined by

$$
\gamma\left(a \otimes \sum_{i} x_{i}\left(g_{i}-1\right)\right)=\sum_{i} a \otimes x_{i}\left(f^{\prime} f\left(g_{i}^{-1}\right) g_{i}\right)[N, N]
$$

for $a \in A, x_{i} \in \mathbb{Z}, 1 \neq g_{i} \in G$. Then $\gamma \beta^{\prime}(a \otimes n[N, N])=\gamma(a \otimes(n-1))=a \otimes n[N, N]$ for each $a \in A, n \in N$, so $\gamma \beta^{\prime}=$ id. It follows that $\operatorname{ker} \beta^{\prime}=0$ and b) is proved.

We recall the notion of relative group (co)homology in the sense of [14]. Let $f$ : $G \rightarrow G^{\prime}$ be a surjective group homomorphism and $A$ an $f$-module. Let $C^{*}(G, A)$ and $C_{*}(G, A)$ be the standard (co)chain complexes for computing group (co)homology. For each $n \geqslant 0$ define

$$
\begin{aligned}
& H^{n}\left(G^{\prime}, G ; A\right)=H^{n} \operatorname{coker}\left(C^{*}\left(G^{\prime}, A\right) \longmapsto C^{*}(G, A)\right) \\
& H_{n}\left(G^{\prime}, G ; A\right)=H_{n} \operatorname{ker}\left(C_{*}(G, A) \rightarrow C_{*}\left(G^{\prime}, A\right)\right) .
\end{aligned}
$$

Notice that this definition differs from the one in [14] by a dimension shift of 1 .

Theorem 7. Let $f: G \rightarrow G^{\prime}$ be a surjective group homomorphism, $A$ an $f$-module. Then

$$
D^{n}\left(\Phi_{f}, A\right) \cong\left\{\begin{array} { l l l } 
{ \operatorname { D e r } ( f , A ) } & { n = 0 } \\
{ H ^ { n + 1 } ( G ^ { \prime } , G ; A ) } & { n > 0 }
\end{array} \quad D _ { n } ( \Phi _ { f } , A ) \cong \left\{\begin{array}{ll}
\operatorname{Diff}(f, A) & n=0 \\
H_{n+1}\left(G^{\prime}, G ; A\right) & n>0
\end{array}\right.\right.
$$

Proof. Let $\mathbb{G}_{\bullet} \Phi_{f}=\left(T_{\bullet}, G_{\bullet}, i_{\bullet}\right), S_{\bullet}=G_{\bullet} / i_{\bullet}\left(T_{\bullet}\right)$ and $\psi_{\bullet}: G_{\bullet} \rightarrow S_{\bullet}$ be the quotient maps. Since $\Phi_{f}$ is aspherical, from $[4] T_{\bullet} \rightarrow \operatorname{ker} f, G_{\bullet} \rightarrow G, S_{\bullet} \rightarrow G^{\prime}$ are free simplicial resolutions and there is a short exact sequence of free simplicial groups $T_{\bullet} \longmapsto G_{\bullet} \rightarrow S_{\bullet}$. Let $\perp$ be the free cotriple on Groups and $\perp_{\bullet} f: \perp_{\bullet} G \rightarrow \perp_{\bullet} G^{\prime}$ 
the induced homomorphisms. Since, for each $n, \psi_{n}$ and $\perp_{n} f$ have a section, from Lemma 6 there is a commutative diagram of cochain complexes

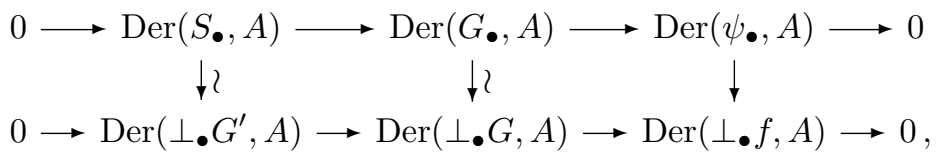

where $\sim$ are cochain homotopy equivalences. Taking the corresponding long exact cohomology sequences in each row and applying the five Lemma we deduce that

$$
H^{n} \operatorname{Der}\left(\psi_{\bullet}, A\right) \cong H^{n} \operatorname{Der}\left(\perp_{\bullet} f, A\right)
$$

for each $n \geqslant 0$. On the other hand there is a commutative diagram of cochain complexes

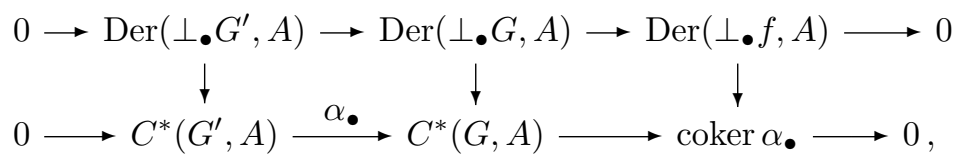

where $\operatorname{Der}\left(\perp_{\bullet} G, A\right) \rightarrow C^{*}(G, A)$ and $\operatorname{Der}\left(\perp_{\bullet} G^{\prime}, A\right) \rightarrow C^{*}\left(G^{\prime}, A\right)$ are the natural cochain maps of the Barr-Beck theory which induce isomorphisms in cohomology (see $[\mathbf{2}])$. Taking the long exact cohomology sequence in each row of the above diagram and applying the five Lemma we deduce that for each $n>0$

$$
H^{n} \operatorname{Der}\left(\perp_{\bullet} f, A\right) \cong H^{n+1} \text { coker } \alpha_{\bullet}=H^{n+1}\left(G, G^{\prime} ; A\right) .
$$

The argument for homology is similar.

\section{Further properties of the (co)homology}

\subsection{The relationship with CCG (co)homology.}

In the next proposition we establish the relationship between the (co)homology theory defined in Section 3 and the one in [4]. For a crossed module $(T, G, \mu)$ let $\zeta(T, G, \mu)=T$.

Proposition 8. Let $\Phi=(T, G, \mu)$ be a crossed module, $A$ a trivial $\pi_{1}$-module. Then for each $n \geqslant 0$

$$
D^{n}(\Phi, A) \cong H_{C C G}^{n+1}((T, G, \mu),(A, 1,0)) .
$$

Suppose further that $A \cong \mathbb{Z}$. Then for each $n \geqslant 0$

$$
D_{n}(\Phi, \mathbb{Z}) \cong \zeta H_{n+1}^{C C G}(T, G, \mu) .
$$

Proof. Let $\mathbb{G}_{\bullet}(T, G, \mu)=\left(T_{\bullet}, G_{\bullet}, i_{\bullet}\right), \quad S_{\bullet}=G_{\bullet} / i_{\bullet}\left(T_{\bullet}\right)$. Since the action of $\pi_{1}$ on $A$ is trivial, using Lemma 6 and the well known isomorphism $T_{n} /\left[G_{n}, T_{n}\right] \cong$ 
$\mathbb{Z} \otimes_{\mathbb{Z} S_{n}}\left(T_{n}\right)_{a b}$, we obtain for each $n \geqslant 0$

$$
\begin{aligned}
& H_{C C G}^{n+1}((T, G, \mu),(A, 1,0))=H^{n} \operatorname{Hom}_{\mathbf{A b C M}}\left(\left(\frac{T_{\bullet}}{\left[G_{\bullet}, T_{\bullet}\right]}, \frac{G_{\bullet}}{\left[G_{\bullet}, G_{\bullet}\right]}, i_{\bullet}\right),(A, 1,0)\right) \cong \\
& \cong H^{n} \operatorname{Hom}_{\mathbb{Z}}\left(\frac{T_{\bullet}}{\left[G_{\bullet}, T_{\bullet}\right]}, A\right) \cong H^{n} \operatorname{Hom}_{\mathbb{Z}}\left(\mathbb{Z} \otimes_{\mathbb{Z} \pi_{1}} \mathbb{Z}_{\pi_{1} \otimes_{\mathbb{Z} S}}\left(T_{\bullet}\right)_{a b}, A\right) \cong \\
& \cong H^{n} \operatorname{Hom}_{\mathbb{Z} \pi_{1}}\left(\operatorname{Diff} \mathbb{G}_{\bullet} \Phi, A\right)=D^{n}(\Phi, A) . \\
& D_{n}(\Phi, \mathbb{Z})=H_{n}\left(\mathbb{Z} \otimes_{\mathbb{Z} \pi_{1}} \operatorname{Diff} \mathbb{G}_{\bullet} \Phi\right) \cong H_{n}\left(\mathbb{Z} \otimes_{\mathbb{Z} \pi_{1}} \mathbb{Z} \pi_{1} \otimes_{\mathbb{Z} S}\left(T_{\bullet}\right)_{a b}\right) \cong H_{n}\left(\frac{T_{\bullet}}{\left[G_{\bullet}, T_{\bullet}\right]}\right) \\
& \cong H_{n}\left(\zeta\left(\frac{T_{\bullet}}{\left[G_{\bullet}, T_{\bullet}\right]}, \frac{G_{\bullet}}{\left[G_{\bullet}, G_{\bullet}\right]}, i \bullet\right)\right) \cong \zeta H_{n}\left(T_{\bullet}, G_{\bullet}, i_{\bullet}\right)_{a b}=\zeta H_{n+1}^{C C G}(T, G, \mu) .
\end{aligned}
$$

\subsection{Interpretation of the first and second cohomology group.}

Let $\Phi=(T, G, \mu)$ be a crossed module acting on the abelian group $A$. We shall need a notion of singular and two-fold special extensions of $(T, G, \mu)$ by $(A, 1,0)$.

Definition 9. Let $\Phi=(T, G, \mu)$ be a crossed module acting on an abelian group $A$.

i) A singular extension of $(T, G, \mu)$ by $(A, 1,0)$ is a short exact sequence of crossed modules

$$
(A, 1,0) \longmapsto\left(T^{\prime}, G, \mu^{\prime}\right) \stackrel{\left(f, \text { id }_{G}\right)}{\rightarrow}(T, G, \mu)
$$

such that the corresponding short exact sequence of cat $^{1}$-groups

$$
(A \times 1,0,0) \longmapsto\left(T^{\prime} \rtimes G, d^{\prime}, s^{\prime}\right) \stackrel{\left(f, \operatorname{id}_{G}\right)}{\rightarrow}(T \rtimes G, d, s)
$$

is a singular extension of $(T \rtimes G, d, s)$ by the $(T \rtimes G, d, s)$-module $(A \times 1,0,0)$ in the sense of categories of interest [21].

ii) $\quad$ 2-fold special extension of $(T, G, \mu)$ by $(A, 1,0)$ is an exact sequence of crossed modules

$$
(A, 1,0) \stackrel{i}{\longrightarrow}\left(T^{\prime \prime}, G^{\prime \prime}, \mu^{\prime \prime}\right) \stackrel{(\alpha, \beta)}{\rightarrow}\left(T^{\prime}, G^{\prime}, \mu^{\prime}\right) \stackrel{(f, r)}{\rightarrow}(T, G, \mu)
$$

such that the corresponding exact sequence of cat ${ }^{1}$-groups

$$
(A \times 1,0,0) \stackrel{i}{\longrightarrow}\left(T^{\prime \prime} \rtimes G^{\prime \prime}, d^{\prime \prime}, s^{\prime \prime}\right) \stackrel{(\alpha, \beta)}{\longrightarrow}\left(T^{\prime} \rtimes G^{\prime}, d^{\prime}, s^{\prime}\right) \stackrel{(f, r)}{\rightarrow}(T \rtimes G, d, s)
$$

is a 2-fold special extension of $(T \rtimes G, d, s)$ by the $(T \rtimes G, d, s)$-module $(A \times$ $1,0,0)$ in the sense of categories of interest [21].

We now give a more explicit characterization of singular and 2-fold special extensions of $(T, G, \mu)$ by $(A, 1,0)$.

Lemma 10. Let $\Phi=(T, G, \mu)$ be a crossed module acting on the abelian group $A$. 
i) A singular extension of $(T, G, \mu)$ by $(A, 1,0)$ consists of a short exact sequence of crossed modules (9) such that if $f^{\prime}: T \rightarrow T^{\prime}$ is a set map with $f f^{\prime}=\mathrm{id}_{T}$, it is

$$
f^{\prime}(t) a f^{\prime}\left(t^{-1}\right)=a, \quad{ }^{g} a={ }^{[g]} a
$$

for all $g \in G, a \in A$, where $[g]=g \mu(T) \in \pi_{1},{ }^{[g]} a$ is the given $\pi_{1}$-module action on $A$ and ${ }^{g} a$ is given by the crossed module action of $G$ on $T^{\prime}$.

ii) A 2-fold special extension of $(T, G, \mu)$ by $(A, 1,0)$ consists of an exact sequence of crossed modules (11) where

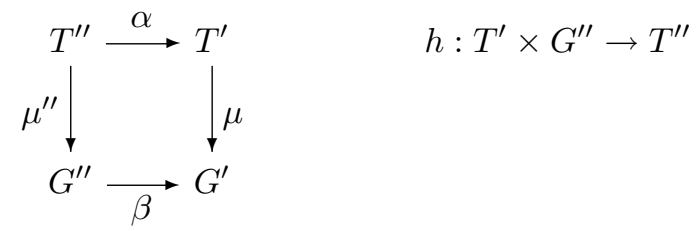

is a crossed square and if $f^{\prime}: T \rightarrow T^{\prime}, \quad r^{\prime}: G \rightarrow G^{\prime}$ are set maps with $f f^{\prime}=\mathrm{id}_{T}, r r^{\prime}=\mathrm{id}_{G}$, then for all $g \in G, t \in T, a \in A$

$$
{ }^{r^{\prime}(g)} a={ }^{[g]} a, \quad f^{\prime}(t) a=a .
$$

Here $[g]=g \mu(T) \in \pi_{1},{ }^{[g]} a$ is the given $\pi_{1}$-module action on $A$ while ${ }^{r^{\prime}(g)} a$ (resp. $\left.f^{\prime}(t) a\right)$ is the action of $G^{\prime}$ (resp. $\left.T^{\prime}\right)$ on $T^{\prime \prime}$ in the crossed square (14).

Proof.

(i) By definition (9) is a singular extension of crossed modules if and only if the corresponding extension of cat $^{1}$-groups (10) is a singular extension in the sense of categories of interest. By definition this means that the induced action of $(T \rtimes G, d, s)$ on $(A \times 1,0,0)$ coincides with the given action, that is

$$
\left(f^{\prime}(t), g\right)(a, 1)\left(f^{\prime}(t), g\right)^{-1}={ }^{(t, g)}(a, 1) .
$$

An easy calculation shows this is equivalent to

$$
\left(f^{\prime}(t){ }^{g} a f^{\prime}\left(t^{-1}\right), 1\right)=\left({ }^{[g]} a, 1\right) .
$$

Hence for all $t \in T, g \in G, A \in A$,

$$
f^{\prime}(t){ }^{g} a f^{\prime}\left(t^{-1}\right)={ }^{[g]} a .
$$

It is immediate to check that (16) is equivalent to (13).

(ii) By definition (11) is a 2-fold special extension of crossed modules if and only if the corresponding extension of cat $^{1}$-groups (12) is a 2 -fold special extension in the sense of categories of interest. By definition (see [21]) this means that

a) $(A \times 1,0,0)$ is a $(T \rtimes G, d, s)$-module,

b) $\left(\left(T^{\prime \prime} \rtimes G^{\prime \prime}, d^{\prime \prime}, s^{\prime \prime}\right),\left(T^{\prime} \rtimes G^{\prime}, d^{\prime}, s^{\prime}\right),(\alpha, \beta)\right)$ is a crossed module in the category of cat ${ }^{1}$-groups,

c) $(A \times 1,0,0) \stackrel{i}{\rightarrow}\left(T^{\prime \prime} \rtimes G^{\prime \prime}, d^{\prime \prime}, s^{\prime \prime}\right)$ is a morphism of $\left(T^{\prime} \rtimes G^{\prime}, d^{\prime}, s^{\prime}\right)$-structures, where $\left(T^{\prime} \rtimes G^{\prime}, d^{\prime}, s^{\prime}\right)$ acts on $(A \times 1,0,0)$ via $(f, r)$. 
Condition b) and Lemma 1 imply that (14) is a crossed square, and the crossed module action of $T^{\prime} \rtimes G^{\prime}$ on $T^{\prime \prime} \rtimes G^{\prime \prime}$ is given by

$$
\left(t^{\prime}, g^{\prime}\right)\left(t^{\prime \prime}, g^{\prime \prime}\right)=\left({ }^{t^{\prime}}\left(g^{\prime} t^{\prime \prime}\right) h\left(t^{\prime}, g^{\prime} g^{\prime \prime}\right), g^{\prime} g^{\prime \prime}\right) .
$$

It easily checked that condition $\mathrm{c}$ ) is equivalent to requiring that the induced action of $(T \rtimes G, d, s)$ on $(A \times 1,0,0)$ given by ${ }^{\left(f^{\prime}(t), r^{\prime}(g)\right)}(a, 1)$ coincides with the given action which is $\left({ }^{[g]} a, 1\right)$. Hence by (17) we obtain

$$
\left(f^{\prime}(t)\left(r^{\prime}(g) a\right) h\left(f^{\prime}(t), 1\right), 1\right)=\left({ }^{[g]} a, 1\right)
$$

for all $t \in T, g \in G, a \in A$. From the axioms of crossed squares [15] $h\left(f^{\prime}(t), 1\right)=1$, hence the above is equivalent to

$$
f^{\prime}(t)\left({ }^{r^{\prime}(g)} a\right)={ }^{[g]} a
$$

for all $t \in T, g \in G, a \in A$. It is straightforward that (18) is equivalent to (15).

Two singular extensions of $(T, G, \mu)$ by $(A, 1,0)$,

$$
(A, 1,0) \longmapsto\left(T_{i}^{\prime}, G, \mu_{i}^{\prime}\right) \rightarrow(T, G, \mu)
$$

$i=1,2$ are congruent if there is a morphism of crossed modules $\psi:\left(T_{1}^{\prime}, G, \mu_{1}^{\prime}\right) \rightarrow$ $\left(T_{2}^{\prime}, G, \mu_{2}^{\prime}\right)$ such that the following diagram commutes

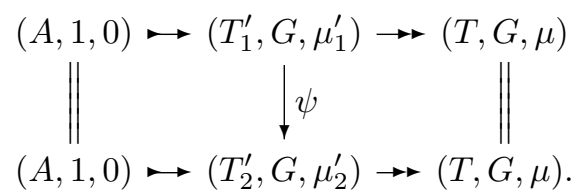

It follows that $\psi$ is an isomorphism. Hence congruence defines an equivalence relation on the set of singular extensions of $\Phi$ by $(A, 1,0)$ and we can consider the set of equivalence classes $\mathcal{E}^{1}(\Phi, A)$. This is an abelian group with Baer sum, the zero element being the class $[(A, 1,0) \longmapsto A \rtimes \Phi \rightarrow \Phi]$.

Two 2-fold special extensions of $\Phi=(T, G, \mu)$ by $(A, 1,0)$ are related if there is a morphism

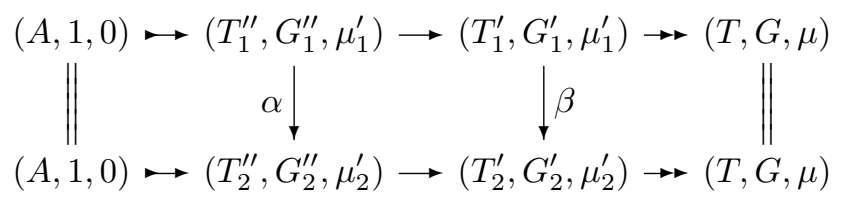

such that $(\alpha, \beta)$ is a morphism of crossed squares. This relation generates an equivalence relation and we denote by $\mathcal{E}^{2}(\Phi, A)$ the set of equivalence classes of 2 -fold special extensions of $\Phi$ by $(A, 1,0)$. This is in fact an abelian group with Baer sum $[21]$.

Proposition 11. Let $\Phi$ be a crossed module acting on the abelian group A. There are isomorphisms of abelian groups

$$
\begin{aligned}
& D^{1}(\Phi, A) \cong \mathcal{E}^{1}(\Phi, A) \\
& D^{2}(\Phi, A) \cong \mathcal{E}^{2}(\Phi, A) .
\end{aligned}
$$


Proof. From Section 2, the cohomology $D^{*}(\Phi, A)$ is cotriple cohomology in the category of interest $\mathbf{C M}$ with coefficients in the $\Phi$-module $(A, 1,0)$. The interpretation in terms of extensions of the first and second cotriple cohomology in any category of interest can be found for example in [21]. The result is thus a direct specialization of [21, Theorem 2.1.3, Proposition 2.1.5, Theorem 2.2.3, Proposition 2.2.4 ].

If $f: G \rightarrow G^{\prime}$ is a surjective group homomorphism and $A$ is an $f$-module, from Theorem 7 and Proposition 11 we deduce that

$$
H^{2}\left(G^{\prime}, G ; A\right) \cong \mathcal{E}^{1}\left(\Phi_{f}, A\right), \quad H^{3}\left(G^{\prime}, G ; A\right) \cong \mathcal{E}^{2}\left(\Phi_{f}, A\right) .
$$

We observe that the first of these isomorphisms recovers a result of [14]. We notice in fact that the group $\mathcal{E}^{1}\left(\Phi_{f}, A\right)$ is isomorphic to the group of relative extensions of $\left(G^{\prime}, G\right)$ by $A$; these consist of exact sequences of groups

$$
0 \rightarrow A \rightarrow M \stackrel{\mu}{\rightarrow} G \stackrel{f}{\rightarrow} G^{\prime} \rightarrow 1
$$

such that $\mu$ is a crossed module and the induced action of $G^{\prime}$ on $A$ coincides with the given one. A congruence of relative extensions is a commutative diagram

$$
\begin{aligned}
& 0 \rightarrow A \rightarrow M \stackrel{\mu}{\rightarrow} G \rightarrow G^{\prime} \rightarrow 1 \\
& 0 \rightarrow A \rightarrow Q \stackrel{\mu^{\prime}}{\rightarrow} G \rightarrow G^{\prime} \rightarrow 1
\end{aligned}
$$

such that $\left(\psi, \mathrm{id}_{G}\right)$ is a morphism of crossed modules. Let $\mathcal{E} x t\left(G^{\prime}, G ; A\right)$ be the set of equivalence classes, made into an abelian group as in [14]. There is a map of abelian groups $\alpha: \mathcal{E} x t\left(G^{\prime}, G ; A\right) \rightarrow \mathcal{E}^{1}(\Phi, A)$

$$
\alpha\left[0 \rightarrow A \rightarrow M \stackrel{\mu}{\rightarrow} G \stackrel{f}{\rightarrow} G^{\prime} \rightarrow 1\right]=[(A, 1,0) \longmapsto(M, G, \mu) \rightarrow(\operatorname{ker} f, G, i)] .
$$

It is immediate to check that $\alpha$ is well defined and that it is a bijection.

The interpretation of $H^{3}\left(G^{\prime}, G ; A\right)$ in terms of equivalence classes of 2-fold special extensions of $(\operatorname{ker} f, G, i)$ by $(A, 1,0)$ does not seem to have been given in the literature as far as the author knows. Notice that the identification of relative group cohomology with cotriple cohomology of a crossed module also allows to give a simplicial interpretation of $H^{n}\left(G, G^{\prime} ; A\right)$ for any $n$ by direct application of the results of $[\mathbf{9}]$.

\subsection{Universal coefficient formulae.}

We shall establish universal coefficient formulae for the (co)homology of a crossed module $\Phi$ acting trivially on an abelian group $A$.

Theorem 12. Let $\Phi=(T, G, \mu)$ be a crossed module acting trivially on an abelian group $A$. Then there are short exact sequences

$$
\begin{gathered}
0 \rightarrow \operatorname{Ext}_{\mathbb{Z}}^{1}\left(D_{n-1}(\Phi, \mathbb{Z}), A\right) \rightarrow D^{n}(\Phi, A) \rightarrow \operatorname{Hom}_{\mathbb{Z}}\left(D_{n}(\Phi, \mathbb{Z}), A\right) \rightarrow 0 \\
0 \rightarrow D_{n}(\Phi, \mathbb{Z}) \otimes_{\mathbb{Z}} A \rightarrow D_{n}(\Phi, A) \rightarrow \operatorname{Tor}_{1}^{\mathbb{Z}}\left(D_{n-1}(\Phi, \mathbb{Z}), A\right) \rightarrow 0 .
\end{gathered}
$$

Proof. Since the action of $\Phi$ on $A$ is trivial, from Proposition 8 we know that $D^{n}(\Phi, A) \cong H^{n+1}(\Phi,(A, 1,0))$ and $D_{n}(\Phi, \mathbb{Z}) \cong \zeta H_{n+1}^{C C G}(\Phi)$. 
Since $\mathbb{Z}$ has global dimension 1 , there exists a resolution $A \rightarrow I^{\bullet}$ of $A$ by injective $\mathbb{Z}$-modules with $I^{m}=0$ for $m \geqslant 2$; then $(A, 1,0) \rightarrow\left(I^{\bullet}, 1,0\right)$ is an injective resolution of $(A, 1,0)$ which satisfies the hypotheses of $[\mathbf{4}$, Theorem 18 (iv) ]. The cohomology universal coefficient sequence then follows from [4, Theorem 18 (iv)].

For the homology case, denote $\Phi_{\bullet}=\mathbb{G}_{\bullet} \Phi$ and let $\varphi_{\bullet \bullet}$ be the double complex of abelian groups

$$
\varphi_{\bullet \bullet}=P_{\bullet} \otimes_{\mathbb{Z} \pi_{1}} \operatorname{Diff} \Phi \bullet
$$

where $P_{\bullet} \rightarrow A$ is a projective $\mathbb{Z}$-resolution of $A$ with $P_{n}=0$ for $n>1$ (such resolution exists since $\mathbb{Z}$ has global dimension 1 ). For any crossed module $\Phi$ acting trivially on $A$, since $\mathbb{Z} \otimes_{\mathbb{Z} \pi_{1}}$ Diff $\Phi \cong D_{0}(\Phi, \mathbb{Z}) \cong \zeta H_{1}^{C C G} \Phi \cong \zeta \Phi_{a b}$ we have

$$
A \otimes_{\mathbb{Z} \pi_{1}} \operatorname{Diff} \Phi \cong A \otimes_{\mathbb{Z}} \zeta \Phi_{a b} .
$$

In particular

$$
P_{\bullet} \otimes_{\mathbb{Z} \pi_{1}} \operatorname{Diff} \Phi \bullet \bullet P \bullet \otimes_{\mathbb{Z}} \zeta\left(\Phi_{\bullet}\right)_{\mathrm{ab}} .
$$

Since $D^{1}\left(\Phi_{q}, A\right)=0$ for each $q$, from the cohomology universal coefficient sequence and from Proposition 8 we obtain $\operatorname{Ext}_{\mathbb{Z}}^{1}\left(\zeta\left(\Phi_{q}\right)_{a b}, A\right)=0$ for every abelian group $A$. It follows that $\zeta\left(\Phi_{q}\right)_{a b}$ is a projective $\mathbb{Z}$-module. Therefore

$$
\begin{aligned}
& H_{p}^{v}\left(P_{\bullet} \otimes_{\mathbb{Z} \pi_{1}} \text { Diff } \Phi_{q}\right) \cong H_{p}^{v}\left(P \bullet \otimes_{\mathbb{Z}} \zeta\left(\Phi_{q}\right)_{\mathrm{ab}}\right) \cong \\
& \cong \operatorname{Tor}_{p}^{\mathbb{Z}}\left(\zeta\left(\Phi_{q}\right)_{\mathrm{ab}}, A\right) \cong \begin{cases}A \otimes_{\mathbb{Z} \pi_{1}} \text { Diff } \Phi_{q} & p=0, \\
0 & p>0 .\end{cases}
\end{aligned}
$$

Taking homology again we obtain

$$
H_{q}^{h} H_{p}^{v}\left(P \bullet \otimes_{\mathbb{Z} \pi_{1}} \operatorname{Diff} \Phi \bullet\right) \cong \begin{cases}D_{q}(\Phi, A) & p=0 \\ 0 & p>0 .\end{cases}
$$

Therefore the spectral sequence

$$
H_{q}^{h} H_{p}^{v}\left(\varphi_{\bullet \bullet}\right) \Rightarrow H_{p+q} \operatorname{Tot} \varphi_{\bullet \bullet}
$$

collapses, giving $H_{n} \operatorname{Tot} \varphi_{\bullet \bullet}=D_{n}(\Phi, A) \quad n \geqslant 0$. Consider the second spectral sequence

$$
H_{q}^{v} H_{p}^{h}\left(\varphi_{\bullet \bullet}\right) \Rightarrow H_{p+q} \operatorname{Tot} \varphi_{\bullet \bullet} .
$$

Fixing $p$ and taking homology we have:

$$
\begin{aligned}
H_{q}^{h}\left(P_{p} \otimes_{\mathbb{Z} \pi_{1}} \operatorname{Diff} \Phi_{\bullet}\right) & \cong H_{q}^{h}\left(P_{p} \otimes_{\mathbb{Z}} \zeta\left(\Phi_{\bullet}\right)_{\mathrm{ab}}\right) \cong P_{p} \otimes_{\mathbb{Z}} H_{q}^{h} \zeta\left(\Phi_{\bullet}\right)_{\mathrm{ab}} \cong \\
& \cong P_{p} \otimes_{\mathbb{Z}} \zeta H_{q+1}^{C C G}(\Phi) \cong \begin{cases}P_{p} \otimes_{\mathbb{Z}} D_{q}(\Phi, \mathbb{Z}) & p=0,1, \\
0 & p>1 .\end{cases}
\end{aligned}
$$

Taking homology again:

$$
H_{p}^{v} H_{q}^{h}\left(P \bullet \otimes_{\mathbb{Z} \pi_{1}} \operatorname{Diff} \Phi \bullet\right)= \begin{cases}\operatorname{Tor}_{p}^{\mathbb{Z}}\left(D_{q}(\Phi, \mathbb{Z}), A\right) & p=0,1, \\ 0 & p>1 .\end{cases}
$$

So we obtain a universal coefficient spectral sequence

$$
E_{p q}^{2} \Rightarrow D_{p+q}(\Phi, A)
$$


which has $E_{p q}^{2}=0$ for $p \neq 0,1$. i.e.

Therefore there are short exact sequences $0 \rightarrow E_{0 n}^{2} \rightarrow H_{n} \operatorname{Tot} \varphi_{\bullet \bullet} \rightarrow E_{1, n-1}^{2} \rightarrow 0$,

$$
0 \rightarrow D_{n}(\Phi, \mathbb{Z}) \otimes_{\mathbb{Z}} A \rightarrow D_{n}(\Phi, A) \rightarrow \operatorname{Tor}_{1}^{\mathbb{Z}}\left(D_{n-1}(\Phi, \mathbb{Z}), A\right) \rightarrow 0
$$

\section{The relationship with the (co)homology of the classifying space.}

In [11] the (co)homology of a crossed module $\Phi$ with coefficients in a $\pi_{1}$-module $A$ is defined as the (co)homology of the classifying space $B(\Phi)$ of the crossed module with coefficients in the local system corresponding to $A$. We recall the algebraic description of this (co)homology. This is a special case of a more general construction, which is well known.

If $G_{*}$ is a simplicial group and $A$ is a $\pi_{0}\left(G_{*}\right)$-module, since $\pi_{1} B G_{*} \cong \pi_{0} G_{*}, A$ is a local system on the classifying space $B G_{*}$ of $G_{*}$. There is an algebraic description of the (co)homology $H_{*}\left(B G_{*}, A\right)$ and $H^{*}\left(B G_{*}, A\right)$. If $C_{*}(G, A)$ (resp. $C^{*}(G, A)$ ) is the standard chain (resp. cochain) complex for computing group homology (resp. cohomology) then there are isomorphisms (see for instance [8, Lemma 5.1]):

$$
H_{*}\left(\operatorname{Tot}\left(C_{*}\left(G_{*}, A\right)\right) \cong H_{*}\left(B G_{*}, A\right), \quad H^{*}\left(\operatorname{Tot}\left(C^{*}\left(G_{*}, A\right)\right) \cong H^{*}\left(B G_{*}, A\right) .\right.\right.
$$

If $G_{*} \rightarrow H_{*}$ is a map of simplicial groups which is a weak equivalence, that is such that it induces isomorphisms of homotopy groups, and $A$ a $\pi_{0}\left(G_{*}\right)$-module, then the induced maps $H_{*}\left(B G_{*}, A\right) \rightarrow H_{*}\left(B H_{*}, A\right)$ and $H^{*}\left(B G_{*}, A\right) \rightarrow H^{*}\left(B H_{*}, A\right)$ are isomorphisms.

Let $N_{*}^{-1}(T, G, \mu)$ be the simplicial group whose Moore complex has length 1 corresponding to the crossed module $\Phi=(T, G, \mu)$ as in Section 1. Taking $G_{*}=$ $N_{*}^{-1}(T, G, \mu)$ in (19) we obtain the algebraic description of the (co)homology of the classifying space of the crossed module. If a morphism of crossed modules $\alpha: \Phi \rightarrow \Phi^{\prime}$ is a weak equivalence then the (co)homology groups of the classifying spaces of $\Phi$ and $\Phi^{\prime}$ with coefficients in a $\pi_{1}$-module $A$ are isomorphic.

Our main result in this section is that the (co)homology of crossed modules defined in Section 2 is related by a long exact sequence to the (co)homology of the classifying space of the crossed module. In proving this result, we also give a simplicial description of the (co)homology of the classifying space. An application of this will be given in Section 7.

In the second part of this section we give an alternative description of the (co)homology $D_{*}(\Phi, A)$ and $D^{*}(\Phi, A)$ without using cotriples.

Theorem 13. Let $\Phi=(T, G, \mu)$ be a crossed module, $A$ a $\pi_{1}$-module. Let $\mathbb{G}_{\bullet} \Phi=$ $\left(T_{\bullet}, G_{\bullet}, \mu_{\bullet}\right)$ and $S_{\bullet} \cong G_{\bullet} / \mu_{\bullet}\left(T_{\bullet}\right)$. Then

i) $\quad B S \bullet$ and $B \Phi$ are weakly homotopy equivalent. 
ii)

$$
\begin{array}{r}
H_{n} \operatorname{Diff}\left(S_{\bullet}, A\right) \cong \begin{cases}H_{n+1}(B \Phi, A) & n>0, \\
A \otimes_{\mathbb{Z} \pi_{1}} \mathfrak{I}_{\pi_{1}} & n=0 .\end{cases} \\
H^{n} \operatorname{Der}\left(S_{\bullet}, A\right) \cong \begin{cases}H^{n+1}(B \Phi, A) & n>0, \\
\operatorname{Der}\left(\pi_{1}, A\right) & n=0,\end{cases}
\end{array}
$$

iii) There are long exact (co)homology sequences

$$
\begin{aligned}
& \cdots \rightarrow D_{n}(\Phi, A) \rightarrow H_{n+1}(G, A) \rightarrow H_{n+1}(B(T, G, \mu), A) \rightarrow \\
& \rightarrow D_{n-1}(\Phi, A) \rightarrow \cdots \rightarrow A \otimes_{\mathbb{Z} \pi_{1}} \operatorname{Diff} \Phi \rightarrow A \otimes_{\mathbb{Z} \pi_{1}} \operatorname{Diff} G \rightarrow \\
& \rightarrow A \otimes_{\mathbb{Z} \pi_{1}} \operatorname{Diff} \pi_{1} \rightarrow 0 \\
& 0 \rightarrow \operatorname{Der}\left(\pi_{1}, A\right) \rightarrow \operatorname{Der}(G, A) \rightarrow \operatorname{Der}(\Phi, A) \rightarrow H^{2}(B(T, G, \mu), A) \rightarrow \\
& \rightarrow H^{2}(G, A) \rightarrow D^{1}(\Phi, A) \rightarrow \cdots
\end{aligned}
$$

\section{Proof.}

(i) Let $s \mathbf{C M}$ be the category of simplicial crossed modules, SimplSet (resp. Simpl ${ }^{2}$ Set) the category of simplicial (resp. bisimplicial) sets. Let $N_{*}^{-1}: \mathbf{C M} \rightarrow$ $\mathbf{S G}_{\leqslant 1}$ be as in $\S 1.1$ and let $\mathcal{N}: \mathbf{S G} \rightarrow$ SimplSet be the functor associating to a simplicial group the diagonal of the bisimplicial set obtained by forming the nerve of the group in each dimension of the simplicial group. The composite $\mathcal{N} \circ N_{*}^{-1}$ is a functor $\mathbf{C M} \rightarrow$ SimplSet. By definition, the classifying space of a crossed module $\Phi$ is the geometric realization of the simplicial set $\mathcal{N} N_{*}^{-1}(\Phi)$. Given a simplicial crossed module, we can apply $\mathcal{N} \circ N_{*}^{-1}$ in each dimension to obtain a bisimplicial set. Hence we have a functor $F: s \mathbf{C M} \rightarrow \operatorname{Simpl}^{2}$ Set.

Consider in particular the simplicial crossed modules $\mathbb{G}_{\bullet} \Phi=\left(T_{\bullet}, G_{\bullet}, \mu_{\bullet}\right)$ and $\Phi=(T, G, \mu)$ (the second is a constant simplicial crossed module).

We claim that $F\left(\mathbb{G}_{\bullet} \Phi\right) \rightarrow F(\Phi)$ is a pointwise weak equivalence of bisimplicial sets in the sense that all the maps $F\left(\mathbb{G}_{\bullet} \Phi\right)_{* m} \rightarrow F(\Phi)_{* m}$ are weak equivalences of simplicial sets. In fact, denoting by $\{\cdot\}$ the one point set

$$
\begin{array}{r}
\left(F \mathbb{G}_{\bullet} \Phi\right)_{n m}=\left(\mathcal{N} N_{*}^{-1}\left(T_{n}, G_{n}, \mu_{n}\right)\right)_{m}= \begin{cases}\{\cdot\} & m=0, \\
T_{n}^{m^{2}} \times G_{n}^{m} & m>0 .\end{cases} \\
(F \Phi)_{n m}=\left(\mathcal{N} N_{*}^{-1}(T, G, \mu)\right)_{m}= \begin{cases}\{\cdot\} & m=0, \\
T^{m^{2}} \times G^{m} & m>0 .\end{cases}
\end{array}
$$

By the properties of the cotriple resolution $\mathbb{G}_{\bullet} \Phi[4], G_{\bullet} \rightarrow G$ and $T_{\bullet} \rightarrow T$ are free simplicial resolutions of groups, therefore $T_{\bullet}^{m^{2}} \times G_{\bullet}^{m} \rightarrow T^{m^{2}} \times G^{m}$ is a weak equivalence in SimplSet, hence for each $m, F\left(\mathbb{G}_{\bullet} \Phi\right)_{* m} \rightarrow F(\Phi)_{* m}$ is a weak equivalence of simplicial sets, that is $F\left(\mathbb{G}_{\bullet} \Phi\right) \rightarrow F(\Phi)$ is a pointwise weak equivalence in $\mathrm{Simpl}^{2}$ Set. It is proved in [12, Ch. IV, Proposition 1.7] that if $f: X \rightarrow Y$ is a pointwise weak equivalence of bisimplicial sets, in the sense that all the maps $f: X_{m} \rightarrow Y_{m}$ are weak equivalences of simplicial sets, then the induced map 
$f_{*}: \operatorname{diag}(X) \rightarrow \operatorname{diag}(Y)$ of associated diagonal simplicial sets is a weak equivalence. It follows that $\operatorname{diag} F\left(\mathbb{G}_{\bullet} \Phi\right) \rightarrow \operatorname{diag} F(\Phi)$ is a weak equivalence in SimplSet, so that the respective geometric realizations $\left|\operatorname{diag} F\left(\mathbb{G}_{\bullet} \Phi\right)\right|$ and $|\operatorname{diag} F(\Phi)|$ are weakly homotopy equivalent.

Recall (see for instance $[\mathbf{2 0}$, p. 94]) that the geometric realization $|\operatorname{diag} X|$ of the diagonal of a bisimplicial set $X$ is homeomorphic to the geometric realization of the simplicial space obtained by taking the geometric realization in vertical directions and is also homeomorphic to the geometric realization of the simplicial space obtained by taking the geometric realization in the horizontal directions. Hence $\left|\operatorname{diag} F\left(\mathbb{G}_{\bullet} \Phi\right)\right|$ is homeomorphic to the geometric realization of the simplicial space $\left\{\left|\mathcal{N} N_{*}^{-1}\left(T_{n}, G_{n}, \mu_{n}\right)\right|\right\}=\left\{B\left(T_{n}, G_{n}, \mu_{n}\right)\right\}$; but since each crossed module $\left(T_{n}, G_{n}, \mu_{n}\right)$ is aspherical, $B\left(T_{n}, G_{n}, \mu_{n}\right) \cong B\left(1, S_{n}, i\right) \cong B S_{n}$. The geometric realization of the simplicial space $\left\{B S_{n}\right\}$ is homeomorphic to the geometric realization of the simplicial group $S_{\bullet}, B S_{\bullet}$. So in conclusion $\left|\operatorname{diag} F\left(\mathbb{G}_{\bullet} \Phi\right)\right| \cong B S_{\bullet}$.

On the other hand clearly $|\operatorname{diag} F(\Phi)|=B \Phi$, so that $B \Phi$ and $B S \bullet$ are weakly homotopy equivalent, proving (i).

(ii) It follows from (i) that for each $n \geqslant 0, H_{n}(B \Phi, A) \cong H_{n}\left(B S_{\bullet}, A\right)$. On the other hand we observe that for each $n>1$

$$
H_{n}\left(B S_{\bullet}, A\right) \cong H_{n-1} \operatorname{Diff}\left(S_{\bullet}, A\right)
$$

In fact, we can compute $H_{n}\left(B S_{\bullet}, A\right)$ as indicated in (19). Since $S_{m}$ is free, $0 \rightarrow$ $\mathfrak{I}_{S_{m}} \rightarrow \mathbb{Z} S_{m} \rightarrow \mathbb{Z} \rightarrow 0$ is a free resolution of the trivial $S_{m}$-module $\mathbb{Z}$, hence $C_{\bullet}\left(S_{m}, A\right)$ is the complex $0 \rightarrow A \otimes_{\mathbb{Z} S_{m}} \mathfrak{I}_{S_{m}} \rightarrow A \rightarrow 0$. It follows that $H_{*}\left(B S_{\bullet}, A\right)$ is the total homology of the bicomplex $\psi_{\text {. }}$

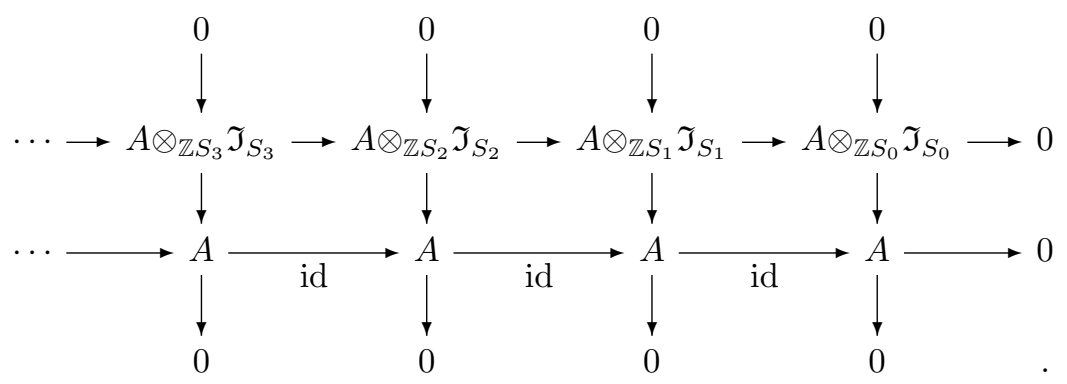

The spectral sequence of this double complex is

$$
E_{p q}^{1}=H_{q}^{h}\left(\psi_{* p}\right)= \begin{cases}0 & p=0, q>0 \text { or } p>1, \\ A & p=0, q=0, \\ H_{q}\left(A \otimes_{\mathbb{Z} S_{\bullet}} \mathfrak{I}_{S_{\bullet}}\right) & p=1, q \geqslant 0 .\end{cases}
$$

Therefore

$$
E_{p q}^{2}= \begin{cases}0 & \text { for } p \neq 0,1 \text { or } p=0, q>0 \\ H_{q}\left(A \otimes_{\mathbb{Z} S_{\bullet} \mathfrak{I}_{S} .}\right) & p=1, q>0 .\end{cases}
$$

So there are short exact sequences $0 \rightarrow E_{0 n}^{2} \rightarrow H_{n} \operatorname{Tot} \psi \bullet \bullet \rightarrow E_{1, n-1}^{2} \rightarrow 0$ and since 
$E_{0 n}^{2}=0$ for $n>0$ and $E_{1, n-1}^{2}=H_{n-1}\left(A \otimes_{\mathbb{Z} S} \mathfrak{I}_{S_{\bullet}}\right)$ for $n>1$ we deduce

$$
H_{n}\left(B S_{\bullet}, A\right) \cong H_{n} \operatorname{Tot} \psi_{\bullet \bullet} \cong E_{1, n-1}^{2}=H_{n-1}\left(A \otimes_{\mathbb{Z} S_{\bullet}} \Im_{S_{\bullet}}\right)
$$

for $n>1$, which is (20). It follows that $H_{n} \operatorname{Diff}\left(S_{\bullet}, A\right) \cong H_{n+1}(B \Phi, A)$ for $n \geqslant 1$.

It remains to prove that $H_{0} \operatorname{Diff}\left(S_{\bullet}, A\right) \cong A \otimes_{\mathbb{Z} \pi_{1}} \mathfrak{I}_{\pi_{1}}$. Consider the following diagram:

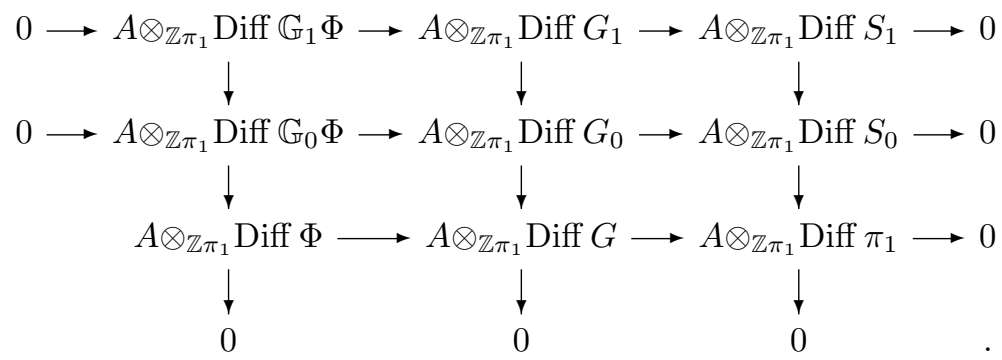

The first two rows from the top are exact by Lemma 6 . We claim that the bottom row is also exact. In fact, it is straightforward to check that there is an exact sequence

$$
0 \rightarrow \operatorname{Der}\left(\pi_{1}, A\right) \stackrel{\alpha}{\rightarrow} \operatorname{Der}(G, A) \stackrel{\beta}{\rightarrow} \operatorname{Der}(\Phi, A)
$$

where $\alpha(D)(g)=D(g \mu(T)), \quad g \in G, \quad D \in \operatorname{Der}\left(\pi_{1}, A\right)$ and $\beta(D)=D d_{1}-D d_{0}$, $d_{1}(t, g)=\mu(t) g, \quad d_{0}(t, g)=g, \quad(t, g) \in T \rtimes G$. Consider the map $\gamma: \operatorname{Diff} \Phi \rightarrow$ $\mathbb{Z} \pi_{1} \otimes_{\mathbb{Z} G} \Im_{G}$ defined by

$$
\gamma\left(x \otimes\left[\sum_{i} a_{i}\left(y_{i}-e\right)\right]\right)=x \otimes\left(\sum_{i} a_{i}\left(d_{1}\left(y_{i}\right)-1\right)-\sum_{i} a_{i}\left(d_{0}\left(y_{i}\right)-1\right)\right)
$$

$x \in \mathbb{Z} \pi_{1}, \quad e \neq y_{i} \in T \rtimes G, \quad a_{i} \in \mathbb{Z}$. By left exactness of $\operatorname{Hom}_{\mathbb{Z} \pi_{1}}(-, A)$ we obtain an exact sequence

$$
0 \rightarrow \operatorname{Hom}_{\mathbb{Z} \pi_{1}}(\operatorname{coker} \gamma, A) \rightarrow \operatorname{Der}(G, A) \rightarrow \operatorname{Der}(\Phi, A) .
$$

Since $\operatorname{Hom}_{\mathbb{Z} \pi_{1}}(\gamma, A)=\beta$, (21) implies that $\operatorname{Hom}_{\mathbb{Z} \pi_{1}}(\operatorname{coker} \gamma, A) \cong \operatorname{Hom}_{\mathbb{Z} \pi_{1}}\left(\mathfrak{I}_{\pi_{1}}, A\right)$ for every $\mathbb{Z} \pi_{1}$-module $A$; hence coker $\gamma \cong \mathfrak{I}_{\pi_{1}}$ and we have the exact sequence

$$
\text { Diff } \Phi \rightarrow \mathbb{Z} \pi_{1} \otimes_{\mathbb{Z} G} \mathfrak{I}_{G} \rightarrow \mathfrak{I}_{\pi_{1}} \rightarrow 0 .
$$

From right exactness of $A \otimes_{\mathbb{Z} \pi_{1}}$ - the claim follows. Since the first two columns from the left of the diagram are also exact, it follows from an easy diagram chasing argument that the third column is also exact. Therefore $H_{0}\left(A \otimes_{\mathbb{Z} \pi_{1}}\right.$ Diff $\left.S \bullet\right) \cong$ $A \otimes_{\mathbb{Z} \pi_{1}}$ Diff $\pi_{1}$. The argument for cohomology is similar.

(iii) From [4] $T_{\bullet} \rightarrow T$ and $G_{\bullet} \rightarrow G$ are free simplicial resolutions and there is a short sequence of free simplicial groups $T_{\bullet} \longmapsto G_{\bullet} \stackrel{\psi_{\bullet}}{\rightarrow} S_{\bullet}$. From Lemma 6 we have short exact sequences of (co)chain complexes

$$
\begin{aligned}
& 0 \rightarrow A \otimes_{\mathbb{Z} \pi_{1}} \operatorname{Diff} \psi \bullet \rightarrow A \otimes_{\mathbb{Z} \pi_{1}} \text { Diff } G_{\bullet} \rightarrow A \otimes_{\mathbb{Z} \pi_{1}} \text { Diff } S \bullet \rightarrow 0 \\
& 0 \rightarrow \operatorname{Der}\left(S_{\bullet}, A\right) \rightarrow \operatorname{Der}\left(G_{\bullet}, A\right) \rightarrow \operatorname{Der}\left(\psi_{\bullet}, A\right) \rightarrow 0 .
\end{aligned}
$$


Taking the corresponding long exact (co)homology sequences and using (ii) we obtain

$$
\begin{aligned}
& \cdots \rightarrow D_{n}(\Phi, A) \rightarrow H_{n+1}(G, A) \rightarrow H_{n} \operatorname{Diff}\left(S_{\bullet}, A\right) \rightarrow D_{n-1}(\Phi, A) \rightarrow \cdots \\
& \rightarrow A \otimes_{\mathbb{Z} \pi_{1}} \operatorname{Diff} \Phi \rightarrow A \otimes_{\mathbb{Z} \pi_{1}} \operatorname{Diff} G \rightarrow H_{0} \operatorname{Diff}\left(S_{\bullet}, A\right) \rightarrow 0 \\
& \quad 0 \rightarrow H^{0} \operatorname{Der}\left(S_{\bullet}, A\right) \rightarrow \operatorname{Der}(G, A) \rightarrow \operatorname{Der}(\Phi, A) \rightarrow H^{1} \operatorname{Der}\left(S_{\bullet}, A\right) \rightarrow \\
& \quad \rightarrow H^{2}(G, A) \rightarrow D^{1}(\Phi, A) \rightarrow \cdots
\end{aligned}
$$

Since, from Proposition $8, D_{n}(\Phi, \mathbb{Z}) \cong \zeta H_{n+1}^{C C G}(T, G, \mu)$, the long exact homology sequences of Theorem 13 for the case $A=\mathbb{Z}$ recovers the result of $[\mathbf{1 3}$, Corollary 4$]$ which is established there via a different method. The following are consequences of the theorem above.

Corollary 14. Let $\Phi=(T, G, \mu)$ and $\Phi^{\prime}=\left(T^{\prime}, G^{\prime}, \mu^{\prime}\right)$ be two crossed modules acting on the abelian group $A$. Suppose that there is a weak equivalence $\Phi \rightarrow \Phi^{\prime}$ inducing isomorphisms $H_{*}(G, A) \cong H_{*}\left(G^{\prime}, A\right), H^{*}(G, A) \cong H^{*}\left(G^{\prime}, A\right)$. Then for each $n \geqslant 0$

$$
D_{n}(\Phi, A) \cong D_{n}\left(\Phi^{\prime}, A\right), \quad D^{n}(\Phi, A) \cong D^{n}\left(\Phi^{\prime}, A\right) .
$$

Proof. By hypothesis there exists a crossed module homomorphism $(f, g): \Phi \rightarrow \Phi^{\prime}$ inducing isomorphisms of homotopy groups. Let $\mathbb{G}_{\bullet} \Phi=\left(T_{\bullet}, G_{\bullet}, \mu_{\bullet}\right), \quad \mathbb{G}_{\bullet} \Phi^{\prime}=$ $\left(T_{\bullet}^{\prime}, G_{\bullet}^{\prime}, \mu_{\bullet}^{\prime}\right), \quad S_{\bullet}=G_{\bullet} / \mu_{\bullet}\left(T_{\bullet}\right), \quad S_{\bullet}^{\prime}=G_{\bullet}^{\prime} / \mu_{\bullet}^{\prime}\left(T_{\bullet}^{\prime}\right)$. The homomorphisms $\mathbb{G}_{\bullet}(f, g)$ : $\mathbb{G}_{\bullet} \Phi \rightarrow \mathbb{G}_{\bullet} \Phi^{\prime}$ induce homomorphisms $S_{\bullet} \rightarrow S_{\bullet}^{\prime}$. By Lemma 6 we have a commutative diagram of chain complexes

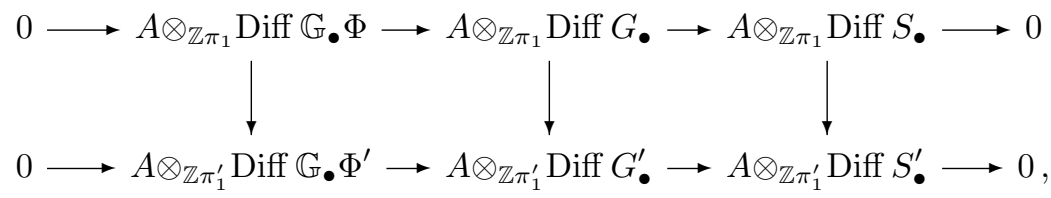

where $\pi_{1}$ and $\pi_{1}^{\prime}$ are the first homotopy groups of $\Phi$ and $\Phi^{\prime}$ respectively. Since $\Phi$ and $\Phi^{\prime}$ are weakly equivalent, by Theorem $13 H_{*}\left(A \otimes_{\mathbb{Z} \pi_{1}}\right.$ Diff $\left.S_{\bullet}\right) \cong H_{*}\left(A \otimes_{\mathbb{Z} \pi_{1}^{\prime}}\right.$ Diff $\left.S_{\bullet}^{\prime}\right)$. Taking the corresponding long exact homology sequence in each row of the above diagram and using the hypothesis that $H_{*}(G, A) \cong H_{*}\left(G^{\prime}, A\right)$ we deduce by the Five Lemma that $D_{*}(\Phi, A) \cong D_{*}\left(\Phi^{\prime}, A\right)$. The case of cohomology is similar.

Corollary 15. Let $\Phi=(T, G, \mu)$ be a crossed module acting on the abelian group $A$, and $\Phi^{\prime}=(R, F, \delta)$ a crossed module weakly equivalent to $\Phi$ with $F$ a free group. Let $\pi_{1} \cong G / \mu(T) \cong F / \delta(R)$. Then

$$
H_{n+2}(B(\Phi), A) \cong D_{n}\left(\Phi^{\prime}, A\right), \quad H^{n+2}(B(\Phi), A) \cong D^{n}\left(\Phi^{\prime}, A\right)
$$

for each $n \geqslant 1$ and

$$
\begin{aligned}
& H_{2}(B(\Phi), A) \cong \operatorname{ker}\left(A \otimes_{\mathbb{Z} \pi_{1}} \text { Diff } \Phi^{\prime} \rightarrow A \otimes_{\mathbb{Z} \pi_{1}} \operatorname{Diff} F\right) \\
& H^{2}(B(\Phi), A) \cong \operatorname{coker}\left(\operatorname{Der}(F, A) \rightarrow \operatorname{Der}\left(\Phi^{\prime}, A\right)\right) .
\end{aligned}
$$


Proof. Apply Theorem 13 using the fact that $H_{*}(B(\Phi), A) \cong H_{*}\left(B\left(\Phi^{\prime}\right), A\right)$, $H^{*}(B(\Phi), A) \cong H^{*}\left(B\left(\Phi^{\prime}\right), A\right)$ and $H_{n}(F, A)=H^{n}(F, A)=0$ for $n \geqslant 2$.

Notice that, since $\operatorname{Der}(\Phi, A) \cong\left\{D \in \operatorname{Hom}_{\mathcal{G} r}(T, A) \mid{ }^{g} D(t)=D\left({ }^{g} t\right)\right\}$ from Corollary 15 we recover the result of [11, Theorem 6], which is established there by different method. Moreover, taking $A=\mathbb{Z}$ and using the fact that $\mathbb{Z} \otimes_{\mathbb{Z} \pi_{1}}$ Diff $\Phi^{\prime} \cong$ $R /[R, F]$ and $\mathbb{Z} \otimes_{\mathbb{Z} \pi_{1}}$ Diff $F \cong F /[F, F]$, we recover the Hopf-type formula for $H_{2}(B(\Phi), \mathbb{Z})$ first proved in $[\mathbf{1 1}$, Theorem 6$]$.

Our aim in the remaining part of this section is to give a description of the (co)homology $D_{*}(\Phi, A)$ and $D^{*}(\Phi, A)$ without using cotriples.

Let $\Phi=(T, G, \mu)$ be a crossed module. The inclusion $(1, G, i) \hookrightarrow(T, G, \mu)$ induces an inclusion of simplicial groups $N_{*}^{-1}(1, G, i) \rightarrow N_{*}^{-1}(T, G, \mu)$. In turn this determines for each $m, n$ an injection $C_{m}\left(N_{n}^{-1}(1, G, i), A\right) \longmapsto C_{m}\left(N_{n}^{-1}(T, G, \mu), A\right)$ and a surjection $C^{m}\left(N_{n}^{-1}(T, G, \mu), A\right) \rightarrow C^{m}\left(N_{n}^{-1}(1, G, i), A\right)$. We therefore have an injective map of chain complexes of abelian groups

$$
\operatorname{Tot} C_{*}\left(N_{*}^{-1}(1, G, i), A\right) \longmapsto \operatorname{Tot} C_{*}\left(N_{*}^{-1}(T, G, \mu), A\right)
$$

and a surjective map of cochain complexes of abelian groups

$$
\operatorname{Tot} C^{*}\left(N_{*}^{-1}(T, G, \mu), A\right) \rightarrow \operatorname{Tot} C^{*}\left(N_{*}^{-1}(1, G, i), A\right) .
$$

Denote by $\beta_{\bullet}(\Phi, A)$ the cokernel in $(24)$ and by $\beta \bullet(\Phi, A)$ the kernel in $(25)$.

Lemma 16. Let $\Phi=(T, G, \mu)$ be an aspherical crossed module with $T, G, \pi_{1}$ free groups, and let $A$ be a $\pi_{1}$-module. Then

$$
\begin{aligned}
& H_{n} \beta_{\bullet}(\Phi, A)=H^{n} \beta^{\bullet}(\Phi, A)=0 \quad \text { for each } n \neq 2, \\
& H_{2} \beta_{\bullet}(\Phi, A) \cong \operatorname{Diff}(\Phi, A), \\
& H^{2} \beta^{\bullet}(\Phi, A) \cong \operatorname{Der}(\Phi, A) .
\end{aligned}
$$

Proof. Consider the long exact homology sequence associated to the short exact sequence of chain complexes

$$
\operatorname{Tot} C_{*}\left(N_{*}^{-1}(1, G, i), A\right) \longmapsto \operatorname{Tot} C_{*}\left(N_{*}^{-1}(T, G, \mu), A\right) \rightarrow \beta_{\bullet}(\Phi, A) .
$$

We have $H_{n}(G, A)=0$ for $n \geqslant 2$ as $G$ is free; since $(T, G, \mu)$ is weakly equivalent to $\left(1, \pi_{1}, i\right)$ and $\pi_{1}$ is free, $H_{n}(B(\Phi), A) \cong H_{n}\left(\pi_{1}, A\right)=0$ for $n \geqslant 2$ and $H_{1}(B(\Phi), A) \cong$ $H_{1}\left(\pi_{1}, A\right)$. Thus this long exact homology sequence gives $H_{n} \beta \bullet(\Phi, A)=H^{n} \beta^{\bullet}(\Phi, A)=$ 0 for $n>2$ and the exact sequence

$$
\begin{aligned}
& 0 \rightarrow H_{2} \beta_{\bullet}(\Phi, A) \rightarrow H_{1}(G, A) \rightarrow H_{1}\left(\pi_{1}, A\right) \rightarrow H_{1} \beta_{\bullet}(\Phi, A) \rightarrow \\
& \rightarrow H_{0}(G, A) \rightarrow H_{0}\left(\pi_{1}, A\right) \rightarrow H_{0} \beta_{\bullet}(\Phi, A) \rightarrow 0 .
\end{aligned}
$$

Since $H_{0}(G, A) \cong A_{G} \cong A_{\pi_{1}} \cong H_{0}\left(\pi_{1}, A\right)$ it follows that $H_{i} \beta_{\bullet}(\Phi, A)=0$ for $i=0,1$ and

$$
H_{2} \beta_{\bullet}(\Phi, A) \cong \operatorname{ker}\left(H_{1}(G, A) \rightarrow H_{1}\left(\pi_{1}, A\right)\right) .
$$

On the other hand, since $\pi_{1}$ is free, the five-term homology sequence associated to the extension $T \longmapsto G \rightarrow \pi_{1}$ reduces to

$$
0 \rightarrow A \otimes_{\mathbb{Z} \pi_{1}} T_{a b} \rightarrow H_{1}(G, A) \rightarrow H_{1}\left(\pi_{1}, A\right) \rightarrow 0 .
$$


Thus by Lemma $6, \quad H_{2} \beta_{\bullet}(\Phi, A) \cong \operatorname{Diff}(\Phi, A)$. The argument for cohomology is similar.

Proposition 17. Let $\Phi$ be a crossed module acting on the abelian group $A$. Then for each $n \geqslant 2$

$$
H_{n}\left(\beta_{\bullet}(\Phi, A)\right) \cong D_{n-2}(\Phi, A), \quad H^{n}\left(\beta^{\bullet}(\Phi, A)\right) \cong D^{n-2}(\Phi, A) .
$$

Proof. Let $\mathbb{G}_{\bullet} \Phi=\left(T_{\bullet}, G_{\bullet}, i_{\bullet}\right)$ and consider the bicomplexes $\left\{\psi_{p q}\right\},\left\{\chi_{p q}\right\},\left\{\mathcal{L}_{p q}\right\}$,

$$
\begin{aligned}
\psi_{p q} & =\beta_{q}\left(\left(T_{p}, G_{p}, i_{p}\right), A\right), \\
\chi_{p q} & =\left(\operatorname{Tot} C_{*}\left(N_{*}^{-1}\left(T_{p}, G_{p}, i_{p}\right), A\right)\right)_{q}, \\
\mathcal{L}_{p q} & =\left(\operatorname{Tot} C_{*}\left(N_{*}^{-1}\left(1, G_{p}, i\right), A\right)\right)_{q} .
\end{aligned}
$$

We aim to show that for each $n \geqslant 2$

$$
H_{n} \operatorname{Tot} \psi_{\bullet \bullet} \cong H_{n} \beta_{\bullet}(\Phi, A) .
$$

The morphism of simplicial crossed modules $\left(T_{\bullet}, G_{\bullet}, i_{\bullet}\right) \rightarrow(T, G, \mu)$ and $\left(1, G_{\bullet}, i\right) \rightarrow$ $(1, G, i)$ (here $(T, G, \mu)$ and $(1, G, i)$ are thought of as constant simplicial crossed modules) induce morphisms of double complexes $\chi_{\bullet \bullet} \rightarrow \operatorname{Tot} C_{*}\left(N_{*}^{-1}(T, G, \mu), A\right)$ and $\mathcal{L}_{\bullet \bullet} \rightarrow$ Tot $C_{*}\left(N_{*}^{-1}(1, G, i), A\right)$. We claim that these morphisms induce isomorphisms in the total homologies in dimensions $n \geqslant 2$. In fact if $S_{\bullet}=G_{\bullet} / i_{\bullet}\left(T_{\bullet}\right)$, the double complex $\chi_{\bullet \bullet}$ gives rise to a spectral sequence

$$
\begin{aligned}
& E_{p q}^{1}=H_{q} \operatorname{Tot} C_{*}\left(N_{*}^{-1}\left(T_{p}, G_{p}, i_{p}\right), A\right) \cong H_{q}\left(B\left(T_{p}, G_{p}, i_{p}\right), A\right) \cong \\
& \cong H_{q}\left(B\left(1, S_{p}, i\right), A\right) \cong H_{q}\left(S_{p}, A\right), \\
& E_{p q}^{2}=H_{p} E_{* q}^{1} .
\end{aligned}
$$

On the other hand since each $S_{p}$ is a free group, the double complex $\alpha \bullet \bullet$

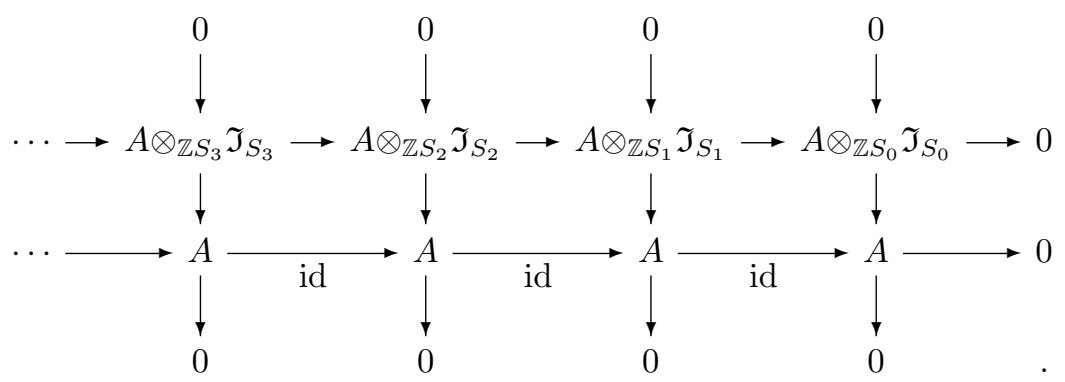

gives rise to a spectral sequence with the same $E^{1}$ and $E^{2}$ terms, hence $H_{n}$ Tot $\alpha_{\bullet \bullet} \cong$ $H_{n} \operatorname{Tot} \chi_{\bullet \bullet}$. As shown in the proof of theorem 13, for each $n \geqslant 2 H_{n}$ Tot $\alpha_{\bullet \bullet}=$ $H_{n-1}\left(A \otimes_{\mathbb{Z} S_{\bullet}} \mathfrak{I}_{S_{\bullet}}\right)$ and by Theorem 13 ii) $H_{n-1}\left(A \otimes_{\mathbb{Z} S_{\bullet}} \mathfrak{I}_{S_{\bullet}}\right) \cong H_{n}(B \Phi, A)$; hence $H_{n} \operatorname{Tot} \chi_{\bullet \bullet} \cong H_{n}(B \Phi, A) \cong H_{n} \operatorname{Tot} C_{*}\left(N_{*}^{-1}(T, G, \mu), A\right)$ for each $n \geqslant 2$.

Similarly, since each $G_{p}$ is free,

$$
\operatorname{Tot} C_{*}\left(N_{*}^{-1}\left(1, G_{\bullet}, i\right), A\right)_{q}= \begin{cases}A & q=0 \\ A \oplus\left(A \otimes_{\mathbb{Z} G_{\bullet}} \mathfrak{I}_{G_{\bullet}}\right) & q>0 .\end{cases}
$$


Therefore $\mathcal{L}_{\bullet \bullet}$ gives rise to a spectral sequence

$$
\begin{gathered}
E_{p q}^{1}=H_{p}\left(\operatorname{Tot} C_{*}\left(N_{*}^{-1}\left(1, G_{\bullet}, i\right), A\right)\right)_{q}= \begin{cases}0 & q=0, \\
H_{p}\left(A \otimes_{\mathbb{Z} G_{\bullet}} \Im_{G_{\bullet}}\right) & q>0 .\end{cases} \\
E_{p q}^{2}=H_{q} E_{p *}^{1}= \begin{cases}0 & q \neq 1, \\
H_{p}\left(A \otimes_{\mathbb{Z} G_{\bullet}} \Im_{G_{\bullet}}\right) & q=1 .\end{cases}
\end{gathered}
$$

It follows that, for each $n \geqslant 2, \quad H_{n} \operatorname{Tot} \mathcal{L}_{\bullet \bullet}=E_{n-1,1}^{2}=H_{n-1}\left(A \otimes_{\mathbb{Z} G_{\bullet}} \Im_{G_{\bullet}}\right)=$ $H_{n}(G, A) \cong H_{n} \operatorname{Tot} C_{*}\left(N_{*}^{-1}(1, G, i), A\right)$. This proves the claim.

Consider the commutative diagram of short exact sequences of double complexes of abelian groups

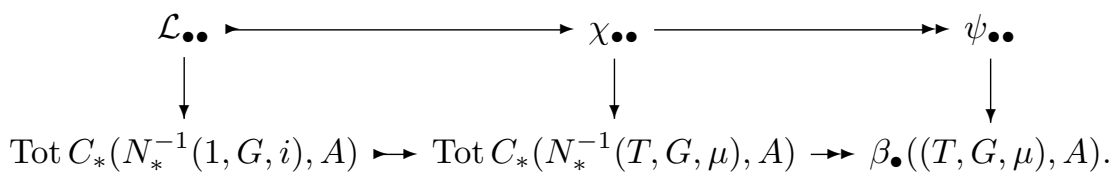

Taking the induced long exact sequences in total homologies in each row of the diagram, from the claim and the Five lemma it follows that, for each $n \geqslant 2$

$$
H_{n} \operatorname{Tot} \psi_{\bullet \bullet} \cong H_{n} \beta_{\bullet}((T, G, \mu), A)
$$

which is (26).

On the other hand, by Lemma 16 the double complex $\psi_{\bullet \bullet}$ gives rise to a spectral sequence

$$
E_{p q}^{1}=H_{q} \beta_{\bullet}\left(\left(T_{p}, G_{p}, i_{p}\right), A\right)= \begin{cases}0 & q \neq 2, \\ \operatorname{Diff}\left(\left(T_{p}, G_{p}, i_{p}\right), A\right) & q=2\end{cases}
$$

so that

$$
E_{p q}^{2}=H_{p} E_{* q}^{1}= \begin{cases}0 & q \neq 2, \\ H_{p} \operatorname{Diff}\left(\left(T_{\bullet}, G_{\bullet}, i_{\bullet}\right), A\right)=D_{p}(\Phi, A) & q=2 .\end{cases}
$$

Hence $E_{p q}^{2} \Rightarrow H_{p+q} \operatorname{Tot} \psi_{\bullet \bullet}=H_{p+q} \beta_{\bullet}((T, G, \mu), A)$ collapses, giving

$$
H_{n} \beta_{\bullet}(\Phi, A)=E_{n-2,2}^{2}=D_{n-2}(\Phi, A)
$$

for $n \geqslant 2$. The argument for cohomology is similar.

We notice that the description of the (co)homology $D^{*}(\Phi, A)$ and $D_{*}(\Phi, A)$ given in the above proposition gives rise to a version of Theorem 13 (ii), which differs from the previous one in low dimensions.

Corollary 18. Let $\Phi=(T, G, \mu)$ be a crossed module, $A$ a $\pi_{1}$-module. There exist long exact (co)homology sequences

$$
\begin{aligned}
& \rightarrow D_{n}(\Phi, A) \rightarrow H_{n+1}(G, A) \rightarrow H_{n+1}(B(T, G, \mu), A) \rightarrow D_{n-1}(\Phi, A) \rightarrow \\
& \cdots \rightarrow H_{2}(G, A) \rightarrow H_{2}(B(T, G, \mu), A) \rightarrow A \otimes_{\mathbb{Z} \pi_{1}} \operatorname{Diff} \Phi \rightarrow \\
& \rightarrow H_{1}(G, A) \rightarrow H_{1}\left(\pi_{1}, A\right) \rightarrow 0
\end{aligned}
$$


Homology, Homotopy and Applications, vol. 5(1), 2003

$$
\begin{aligned}
& 0 \rightarrow H^{1}\left(\pi_{1}, A\right) \rightarrow H^{1}(G, A) \rightarrow \operatorname{Der}(\Phi, A) \rightarrow H^{2}(B(T, G, \mu), A) \rightarrow \\
& \rightarrow H^{2}(G, A) \rightarrow D^{1}(\Phi, A) \rightarrow \cdots
\end{aligned}
$$

Proof. Take the long exact (co)homology sequences associated to the short exact sequences of (co)chain complexes

$$
\begin{aligned}
& \operatorname{Tot} C_{*}\left(N_{*}^{-1}(1, G, i), A\right) \longmapsto \operatorname{Tot} C_{*}\left(N_{*}^{-1}(T, G, \mu), A\right) \rightarrow \beta_{\bullet}(\Phi, A) \\
& \beta^{\bullet}(\Phi, A) \longmapsto \operatorname{Tot} C^{*}\left(N_{*}^{-1}(T, G, \mu), A\right) \rightarrow \operatorname{Tot} C^{*}\left(N_{*}^{-1}(1, G, i), A\right) .
\end{aligned}
$$

Apply Proposition 17 and the fact (see $[\mathbf{1 1}])$ that $H_{1}(B(\Phi), A) \cong H_{1}\left(\pi_{1}, A\right)$ and $H^{1}(B(\Phi), A)=H^{1}\left(\pi_{1}, A\right)$.

We finally notice that a more topological approach than the one given in this paper should allow to obtain a topological interpretation of the (co)homology $D^{*}(\Phi, A)$ and $D_{*}(\Phi, A)$ as relative (co)homology of the pair of spaces $(B \Phi, B G)$ with local coefficients. In fact, it is reasonable to conjecture that, for each $n>0, H^{n} \beta^{\bullet}(\Phi, A)$ (resp. $\left.H_{n} \beta_{\bullet}(\Phi, A)\right)$ is isomorphic to $H^{n}(B \Phi, B G ; A)\left(\right.$ resp. $H_{n}(B \Phi, B G ; A)$ ), so that by Proposition 17 , for each $n \geqslant 2 D^{n-2}(\Phi, A)$ (resp. $D_{n-2}(\Phi, A)$ ) would be isomorphic to $H^{n}(B \Phi, B G ; A)$ (resp. $\left.H_{n}(B \Phi, B G ; A)\right)$.

\section{An example}

Let $M$ be a $G$-module and consider the crossed module $\Phi=(M, G, 0)$. The map of crossed modules $\left(i, \mathrm{id}_{G}\right):(1, G, i) \rightarrow(M, G, 0)$ has a section $\left(0, \operatorname{id}_{G}\right):(M, G, 0) \rightarrow$ $(1, G, i)$. Therefore the corresponding map $B(G) \hookrightarrow B(M, G, 0)$ has a section $B(M, G, 0) \rightarrow B(G)$. Hence the long exact sequences of Theorem 13 give split short exact sequences for each $n \geqslant 2$

$$
\begin{aligned}
& 0 \rightarrow H_{n}(G, A) \leftrightarrows H_{n}(B(M, G, 0), A) \rightarrow D_{n-2}((M, G, 0), A) \rightarrow 0 \\
& 0 \rightarrow D^{n-2}((M, G, 0), A) \rightarrow H^{n}(B(M, G, 0), A) \leftrightarrows H^{n}(G, A) \rightarrow 0
\end{aligned}
$$

It follows that for each $n \geqslant 2$

$$
\begin{aligned}
& H_{n}(B(M, G, 0), A) \cong H_{n}(G, A) \oplus D_{n-2}((M, G, 0), A) \\
& H^{n}(B(M, G, 0), A) \cong H^{n}(G, A) \oplus D^{n-2}((M, G, 0), A) .
\end{aligned}
$$

Recall that for every crossed module $(T, G, \mu)$ with homotopy groups $\pi_{1}$ and $\pi_{2}$ there is a fibration sequence

$$
K\left(\pi_{2}, 2\right) \rightarrow|B(T, G, \mu)| \rightarrow K\left(\pi_{1}, 1\right)
$$

where $K\left(\pi_{2}, 2\right)$ and $K\left(\pi_{1}, 1\right)$ are Eilenberg-MacLane spaces. Hence we have corresponding Serre spectral sequences:

$$
\begin{aligned}
& E_{p q}^{2}=H_{p}\left(\pi_{1}, H_{q}\left(K\left(\pi_{2}, 2\right), A\right)\right) \Rightarrow H_{p+q}(B(T, G, \mu), A) \\
& E_{2}^{p q}=H^{p}\left(\pi_{1}, H^{q}\left(K\left(\pi_{2}, 2\right), A\right)\right) \Rightarrow H^{p+q}(B(T, G, \mu), A) .
\end{aligned}
$$

In the following proposition we shall use the well known fact that, for every abelian group $A$

$$
H_{1} K(A, 2)=0=H_{3} K(A, 2), \quad H_{2} K(A, 2) \cong A, \quad H_{4} K(A, 2) \cong \Gamma^{2} A
$$


where $\Gamma^{2}$ denotes Whitehead's universal quadratic functor.

Proposition 19. Let $M$ be a $\mathbb{Z} G$-module and let $\Phi=(M, G, 0)$ act on the abelian group $A$.

a) There are exact sequences

$$
\begin{aligned}
& D_{2}(\Phi, A) \rightarrow H_{2}\left(G, M \otimes_{\mathbb{Z}} A\right) \rightarrow H_{0}\left(G, \operatorname{Tor}_{1}^{\mathbb{Z}}(M, A)\right) \rightarrow \\
& \quad \rightarrow D_{1}(\Phi, A) \rightarrow H_{1}\left(G, M \otimes_{\mathbb{Z}} A\right) \rightarrow 0 \\
& 0 \rightarrow H^{1}\left(G, \operatorname{Hom}_{\mathbb{Z}}(M, A)\right) \rightarrow D^{1}(\Phi, A) \rightarrow H^{0}\left(G, \operatorname{Ext}_{\mathbb{Z}}^{1}(M, A)\right) \rightarrow \\
& \rightarrow H^{2}\left(G, \operatorname{Hom}_{\mathbb{Z}}(M, A)\right) \rightarrow D^{2}(\Phi, A) .
\end{aligned}
$$

b) If $\operatorname{Tor}_{1}^{\mathbb{Z}}(M, A)=0$ then there is an exact sequence

$$
\begin{aligned}
& D_{3}(\Phi, A) \rightarrow H_{3}\left(G, M \otimes_{\mathbb{Z}} A\right) \rightarrow H_{0}\left(G, \Gamma^{2} M \otimes_{\mathbb{Z}} A\right) \rightarrow \\
& \rightarrow D_{2}(\Phi, A) \rightarrow H_{2}\left(G, M \otimes_{\mathbb{Z}} A\right) \rightarrow 0 .
\end{aligned}
$$

If $\operatorname{Ext}_{\mathbb{Z}}^{1}(M, A)=0$ then there is an exact sequence

$$
\begin{aligned}
0 & \rightarrow H^{2}\left(G, \operatorname{Hom}_{\mathbb{Z}}(M, A)\right) \rightarrow D^{2}(\Phi, A) \rightarrow H^{0}\left(G, \operatorname{Hom}_{\mathbb{Z}}\left(\Gamma^{2} M, A\right)\right) \rightarrow \\
& \rightarrow H^{3}\left(G, \operatorname{Hom}_{\mathbb{Z}}(M, A)\right) \rightarrow D^{3}(\Phi, A) .
\end{aligned}
$$

Proof. The Serre spectral sequences for the crossed module $(M, G, 0)$ are

$$
\begin{aligned}
& E_{p q}^{2}=H_{p}\left(G, H_{q}(K(M, 2), A)\right) \Rightarrow H_{p+q}(B(M, G, 0), A) \\
& E_{2}^{p q}=H^{q}\left(G, H^{q}(K(M, 2), A)\right) \Rightarrow H^{p+q}(B(M, G, 0), A)
\end{aligned}
$$

while the same spectral sequence for the crossed module $(1, G, i)$ has $E_{p q}^{2}=E_{2}^{p q}=0$ for $q \neq 0$; also $H_{n}(B(1, G, i), A)=H_{n}(G, A)$ and $H^{n}(B(1, G, i), A)=H^{n}(G, A)$.

Hence from $(27)$ we deduce that there are spectral sequences

$$
\begin{aligned}
& \widetilde{E}_{p q}^{2}=H_{p}\left(G, H_{q+2}(K(M, 2), A)\right) \Rightarrow D_{p+q}((M, G, 0), A) \\
& \widetilde{E}_{2}^{p q}=H^{p}\left(G, H^{q+2}(K(M, 2), A)\right) \Rightarrow D^{p+q}((M, G, 0), A) .
\end{aligned}
$$

The corresponding exact sequences of low degree terms are

$$
\begin{aligned}
& D_{2}(\Phi, A) \rightarrow H_{2}\left(G, H_{2}(K(M, 2), A)\right) \rightarrow H_{0}\left(G, H_{3}(K(M, 2), A)\right) \rightarrow \\
& \quad \rightarrow D_{1}(\Phi, A) \rightarrow H_{1}\left(G, H_{2}(K(M, 2), A)\right) \rightarrow 0 . \\
& 0 \rightarrow H^{1}\left(G, H^{2}(K(M, 2), A)\right) \rightarrow D^{1}(\Phi, A) \rightarrow H^{0}\left(G, H^{3}(K(M, 2), A)\right) \rightarrow \\
& \rightarrow H^{2}\left(G, H^{2}(K(M, 2), A)\right) \rightarrow D^{2}(\Phi, A) .
\end{aligned}
$$

By (29) and the universal coefficient theorem,

$$
\begin{array}{ll}
H_{2}(K(M, 2), A) \cong M \otimes_{\mathbb{Z}} A, & H_{3}(K(M, 2), A) \cong \operatorname{Tor}_{1}^{\mathbb{Z}}(M, A) \\
H^{2}(K(M, 2), A) \cong \operatorname{Hom}_{\mathbb{Z}}(M, A), & H^{3}(K(M, 2), A) \cong \operatorname{Ext}_{\mathbb{Z}}^{1}(M, A)
\end{array}
$$

so that part a) follows. 
If $\operatorname{Tor}_{1}^{\mathbb{Z}}(M, A)=0$ then $\widetilde{E}_{p 1}^{2}=H_{p}\left(G, \operatorname{Tor}_{1}^{\mathbb{Z}}(M, A)\right)=0$. Hence (see [5]) there is an exact sequence $D_{3} \rightarrow \widetilde{E}_{3,0}^{2} \rightarrow \widetilde{E}_{0,2}^{2} \rightarrow D_{2} \rightarrow \widetilde{E}_{2,0}^{2} \rightarrow 0$, i.e.

$$
\begin{aligned}
& D_{3}(\Phi, A) \rightarrow H_{3}\left(G, H_{2}(K(M, 2), A) \rightarrow H_{0}\left(G, H_{4}(K(M, 2), A)\right) \rightarrow\right. \\
& \rightarrow D_{2}(\Phi, A) \rightarrow H_{2}\left(G, H_{2}(K(M, 2), A)\right) \rightarrow 0 .
\end{aligned}
$$

From (29) and the universal coefficient theorem, we have $H_{4}(K(M, 2), A)=\Gamma^{2} M \otimes_{\mathbb{Z}} A$ so that part b) follows for the homology case.

If $\operatorname{Ext}_{\mathbb{Z}}^{1}(M, A)=0$ then $\widetilde{E}_{2}^{p 1}=H^{p}\left(G, \operatorname{Ext}_{\mathbb{Z}}^{1}(M, A)\right)=0$ so there is an exact sequence $0 \rightarrow \widetilde{E}_{2}^{2,0} \rightarrow D^{2} \rightarrow \widetilde{E}_{2}^{0,2} \rightarrow \widetilde{E}_{2}^{3,0} \rightarrow D^{3}$, i.e.

$$
\begin{aligned}
0 & \rightarrow H^{2}\left(G, H^{2}(K(M, 2), A)\right) \rightarrow D^{2}(\Phi, A) \rightarrow H^{0}\left(G, H^{4}(K(M, 2), A)\right) \rightarrow \\
& \rightarrow H^{3}\left(G, H^{2}(K(M, 2), A)\right) \rightarrow D^{3}(\Phi, A) .
\end{aligned}
$$

From the universal coefficient theorem $\left.H^{4}(K(M, 2), A)\right)=\operatorname{Hom}_{\mathbb{Z}}\left(\Gamma^{2} M, A\right)$ so that part b) follows.

We point out that for the case of homology with $\mathbb{Z}$-coefficients, (28) and the homology exact sequences of Proposition 19 were also given in [13].

\section{An application to the cohomology of the classifying space}

In this section we apply the simplicial description of the cohomology of the classifying space of a crossed module proved in Theorem 13 ii) to give an interpretation of these cohomology groups in dimensions $n=2,3$.

Lemma 20. Let $\Phi=(T, G, \mu)$ be a crossed module, $A$ an abelian group. Then $(1, A, i)$ is a $\Phi$-module if and only if $A$ is a $\pi_{1}$-module. In this case there is an isomorphism

$$
\operatorname{Der}(\Phi,(1, A, i)) \cong \operatorname{Der}\left(\pi_{1}, A\right) .
$$

Proof. From Section 1, the singular object $(1, A, i)$ is a $\Phi$-module if and only if there is a split extension of crossed modules

$$
(1, A, i) \longmapsto\left(T^{\prime}, G^{\prime}, \mu^{\prime}\right) \rightleftarrows(T, G, \mu) .
$$

In particular we have split short exact sequences of groups $1 \longmapsto T^{\prime} \rightarrow T$ and $A \longmapsto G^{\prime} \rightleftarrows G$; thus we can assume that $T^{\prime}=T, \quad G^{\prime} \cong A \rtimes G$, that the map $\operatorname{pr}_{G}: A \rtimes G \rightarrow G$ is $\operatorname{pr}_{G}(a, g)=g$ and that the map $i_{G}: G \rightarrow A \rtimes G$ is $i_{G}(g)=(0, g)$. Hence we have the split short exact sequence

$$
(1, A, i) \longrightarrow\left(T, A \rtimes G, \mu^{\prime}\right) \frac{\left(\mathrm{id}_{T}, i_{G}\right)}{\stackrel{\left(\mathrm{id}_{T}, \mathrm{pr}_{G}\right)}{\longrightarrow}}(T, G, \mu) .
$$

Since $\left(\mathrm{id}_{T}, i_{G}\right)$ is a crossed module map, $\mu^{\prime} \mathrm{id}_{T}=i_{G} \mu$, hence $\mu^{\prime}(t)=(0, \mu(t))$ for all $t \in T$, that is $\mu^{\prime}=(0, \mu)$. Since $\left(\mathrm{id}_{T}, \mathrm{pr}_{G}\right)$ is a crossed module map, ${ }^{(a, g)} t={ }^{g} t$ for all $(a, g) \in A \rtimes G, t \in T$. The axioms of crossed module for $(T, A \rtimes G,(0, \mu))$ give, for all $(a, g) \in A \rtimes G, t \in T$

$$
(0, \mu)\left({ }^{(a, g)} t\right)=(a, g)(0, \mu(t))(a, g)^{-1} .
$$


An easy calculation shows that this is equivalent to

$$
\left(0, \mu\left({ }^{g} t\right)\right)=\left(a-{ }^{\mu\left({ }^{g} t\right)} a, \mu\left({ }^{g} t\right)\right)
$$

for all $t \in T, g \in G, a \in A$. It follows that $a={ }^{\mu\left({ }^{g} t\right)} a$. In particular, taking $g=1$ we obtain $a=\mu(t) a$ for all $t \in T, a \in A$, so that $A$ is a $\pi_{1}$-module.

Conversely, if $A$ is a $\pi_{1}$-module, then (31) is a split singular extension of crossed modules, hence $(1, A, i)$ is a $\Phi$-module. We have

$$
\operatorname{Der}(\Phi,(1, A, i)) \cong \operatorname{Hom}_{\mathbf{C M} / \Phi}(\Phi,(T, A \rtimes G,(0, \mu)) .
$$

We now show that there is an isomorphism

$$
\alpha: \operatorname{Hom}_{\mathbf{C M} / \Phi}\left(\Phi,(T, A \rtimes G,(0, \mu)) \rightarrow \operatorname{Der}\left(\pi_{1}, A\right) .\right.
$$

Let $\alpha\left(\operatorname{id}_{T},\left(D, \operatorname{id}_{G}\right)\right)=D$. Then $D \in \operatorname{Der}(G, A)$ and since $\left(D, \operatorname{id}_{G}\right) \mu=(0, \mu)$ we have $D \mu=0$ so that $D \in \operatorname{Der}\left(\pi_{1}, A\right)$. Clearly $\alpha$ is injective. Given $D \in \operatorname{Der}\left(\pi_{1}, A\right)$ let $\bar{D}(g)=D(g \mu(T)), g \in G$. Then $\bar{D} \in \operatorname{Der}(G, A)$ and $\alpha\left(\operatorname{id}_{T},\left(\bar{D}, \operatorname{id}_{G}\right)\right)=D$, so that $\alpha$ is also surjective.

We can similarly define a functor $\operatorname{Der}(-,(1, A, i)): \mathbf{C M} / \Phi \rightarrow \mathbf{A b}$ on the slice category.

In the next proposition we show that the cohomology of the classifying space of a crossed module can be described as cotriple cohomology.

Proposition 21. Let $\Phi=(T, G, \mu)$ be a crossed module, $A$ a $\pi_{1}$-module. Then for each $n>0$

$$
H^{n}(B(\Phi), A) \cong H^{n-1} \operatorname{Der}\left(\mathbb{G}_{\bullet} \Phi,(1, A, i)\right) .
$$

Proof. Let $\mathbb{G}_{\bullet} \Phi=\left(T_{\bullet}, G_{\bullet}, i_{\bullet}\right), S_{\bullet}=G_{\bullet} / i_{\bullet}\left(T_{\bullet}\right)$. From Lemma 20

$$
H^{n} \operatorname{Der}\left(\mathbb{G}_{\bullet} \Phi,(1, A, i)\right) \cong H^{n} \operatorname{Der}\left(S_{\bullet}, A\right) .
$$

The result follows from Theorem 13 ii).

The following corollary generalizes a result of [4, Theorem 10 (iv)] which is established there in the case of aspherical crossed modules.

Corollary 22. Let $\Phi=(T, G, \mu)$ be a crossed module, $A$ a trivial $\pi_{1}$-module. Then for each $n>0$

$$
H_{C C G}^{n}((T, G, \mu),(1, A, i)) \cong H^{n}(B(\Phi), A) .
$$

Proof. Let $\mathbb{G}_{\bullet} \Phi=\left(T_{\bullet}, G_{\bullet}, i_{\bullet}\right), S_{\bullet}=G_{\bullet} / i_{\bullet}\left(T_{\bullet}\right)$. Since actions are trivial

$$
\operatorname{Der}\left(S_{\bullet}, A\right) \cong \operatorname{Hom}_{\mathcal{G} p}\left(S_{\bullet}, A\right) \cong \operatorname{Hom}_{\mathbf{C M}}\left(\left(T_{\bullet}, G_{\bullet}, i_{\bullet}\right),(1, A, i)\right)
$$

By definition $H_{C C G}^{n}((T, G, \mu),(1, A, i)) \cong H^{n-1} \operatorname{Hom}_{\mathbf{C M}}\left(\left(T_{\bullet}, G_{\bullet}, i_{\bullet}\right),(1, A, i)\right)$ and the result follows from Theorem $13 \mathrm{ii})$.

We finally obtain the interpretation for the second and third cohomology group of the classifying space. We need the notion of singular and 2-fold special extensions of $(T, G, \mu)$ by $(1, A, i)$.

Definition 23. Let $\Phi=(T, G, \mu)$ be a crossed module acting on an abelian group A. 
i) A singular extension of $(T, G, \mu)$ by $(1, A, i)$ is a short exact sequence of crossed modules

$$
(1, A, i) \longmapsto\left(T, G^{\prime}, \mu^{\prime}\right) \stackrel{\left(\mathrm{id}_{T}, f\right)}{\rightarrow}(T, G, \mu)
$$

such that the corresponding short exact sequence of cat ${ }^{1}$-groups

$$
(1 \times A, \mathrm{id}, \mathrm{id}) \longmapsto\left(T \rtimes G^{\prime}, d^{\prime}, s^{\prime}\right) \rightarrow(T \rtimes G, d, s)
$$

is a singular extension of $(T \rtimes G, d, s)$ by the $(T \rtimes G, d, s)$-module $(1 \times A$, id, id) in the sense of categories of interest [21].

ii) $\quad$ 2-fold special extension of $(T, G, \mu)$ by $(1, A, i)$ is an exact sequence of crossed modules

$$
(1, A, i) \stackrel{i}{\longrightarrow}\left(T^{\prime \prime}, G^{\prime \prime}, \mu^{\prime \prime}\right) \stackrel{(\alpha, \beta)}{\longrightarrow}\left(T^{\prime}, G^{\prime}, \mu^{\prime}\right) \stackrel{(f, r)}{\rightarrow}(T, G, \mu)
$$

such that the corresponding exact sequence of cat ${ }^{1}$-groups

$$
(1 \times A, \mathrm{id}, \mathrm{id}) \longmapsto\left(T^{\prime \prime} \rtimes G^{\prime \prime}, d^{\prime \prime}, s^{\prime \prime}\right) \stackrel{(\alpha, \beta)}{\longrightarrow}\left(T^{\prime} \rtimes G^{\prime}, d^{\prime}, s^{\prime}\right) \stackrel{(f, r)}{\rightarrow}(T \rtimes G, d, s)
$$

is a 2-fold special extension of $(T \rtimes G, d, s)$ by the $(T \rtimes G, d, s)$-module $(1 \times$ $A, \mathrm{id}, \mathrm{id})$ in the sense of categories of interest [21].

A more explicit characterization of singular and 2-fold special extensions of $(T, G, \mu)$ by $(1, A, i)$ can be given as follows.

Lemma 24. Let $\Phi=(T, G, \mu)$ be a crossed module acting on the abelian group $A$.

i ) A singular extension of $(T, G, \mu)$ by $(1, A, i)$ consists of a short exact sequence of crossed modules (32) such that if $f^{\prime}: G \rightarrow G^{\prime}$ is a set map with $f f^{\prime}=\mathrm{id}_{G}$, it is $f^{\prime}(g) a f^{\prime}\left(g^{-1}\right)={ }^{[g]}$ a for all $g \in G, a \in A$ where $[g]=g \mu(T) \in \pi_{1}$ and ${ }^{[g]} a$ is the given $\pi_{1}$-module action on $A$.

ii) $\quad$ 2-fold special extension of $(T, G, \mu)$ by $(1, A, i)$ consists of an exact sequence of crossed modules (34) where

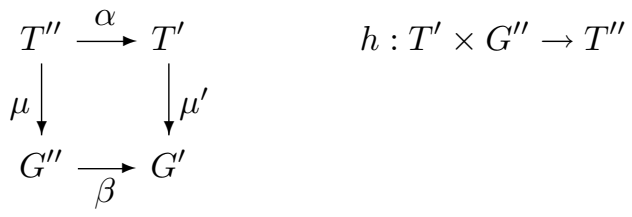

is a crossed square and if $r^{\prime}: G \rightarrow G^{\prime}$ is a set map with $r r^{\prime}=\mathrm{id}_{G}$, then for all $g \in G, a \in A$,

$$
{ }^{\prime}(g) a={ }^{[g]} a .
$$

Here $[g]=g \mu(T) \in \pi_{1},{ }^{[g]} a$ is the given $\pi_{1}$-module action on $A$ and ${ }^{r^{\prime}(g)} a$ is the action of $G^{\prime}$ on $G^{\prime \prime}$ in the crossed square (36).

\section{Proof.}

i) By definition, (33) is a singular extension of cat ${ }^{1}$-groups. Hence for all $t \in T$, $g \in G, a \in A$

$$
\left(t, f^{\prime}(g)\right)(1, a)\left(t, f^{\prime}\left(g^{-1}\right)\right)=\left(1,{ }^{[g]} a\right)
$$


that is

$$
\left(t^{f^{\prime}(g) a f^{\prime}\left(g^{-1}\right)} t^{-1}, f^{\prime}(g) a f^{\prime}\left(g^{-1}\right)\right)=\left(1,{ }^{[g]} a\right) .
$$

Since $(1, A, i)$ is a normal subcrossed module of $(T, G, \mu), t^{f^{\prime}(g) a f^{\prime}\left(g^{-1}\right)} t^{-1}=1$ for all $t \in T, g \in G, a \in A$; hence we only require that $f^{\prime}(g) a f^{\prime}\left(g^{-1}\right)={ }^{[g]} a$.

ii) By definition, (34) is a 2-fold extension in cat ${ }^{1}$-groups. Let $f^{\prime}: T \rightarrow T^{\prime}$ be a set map with $f f^{\prime}=\mathrm{id}_{T}$. Since $\left(\left(T^{\prime \prime} \rtimes G^{\prime \prime}, d^{\prime \prime}, s^{\prime \prime}\right),\left(T^{\prime} \rtimes G^{\prime}, d^{\prime}, s^{\prime}\right),(\alpha, \beta)\right)$ is a crossed module in the category of cat ${ }^{1}$-groups, by Lemma 1 (36) is a crossed square and the crossed module action of $T^{\prime} \rtimes G^{\prime}$ on $T^{\prime \prime} \rtimes G^{\prime \prime}$ is given by

$$
{ }^{\left(t^{\prime}, g^{\prime}\right)}\left(t^{\prime \prime}, g^{\prime \prime}\right)=\left(t^{\prime}\left(g^{\prime} t^{\prime \prime}\right) h\left(t^{\prime}, g^{\prime} g^{\prime \prime}\right), g^{\prime} g^{\prime \prime}\right) .
$$

Further, arguing as in the proof of Lemma $10 \mathrm{ii})$, the induced action of $(T \rtimes$ $G, d, s)$ on $\left(1 \times A\right.$, id, id) given by ${ }^{\left(f^{\prime}(t), r^{\prime}(g)\right)}(1, a)$ has to coincide with the given action, which is $\left(1,{ }^{[g]} a\right)$. Hence by $(37)$ we obtain

$$
\left(h\left(f^{\prime}(t),{ }^{r^{\prime}(g)} a\right),{ }^{r^{\prime}(g)} a\right)=\left(1,{ }^{[g]} a\right)
$$

for all $t \in T, g \in G, a \in A$. By the axioms of crossed squares [15]

$$
\alpha h\left(f^{\prime}(t), r^{r^{\prime}(g)} a\right)=f^{\prime}(t)^{\beta\left(r^{r^{\prime}(g)} a\right)} f^{\prime}(t)^{-1}=f^{\prime}(t) f^{\prime}(t)^{-1}=1 .
$$

Hence, since $\alpha$ is injective, $h\left(f^{\prime}(t),{ }^{\prime}(g) a\right)=1$. Therefore we only require

$$
{ }^{\prime}(g) a={ }^{[g]} a
$$

for all $g \in G, a \in A$.

It is possible to introduce an equivalence relation on the set of singular and 2-fold special extensions of $(T, G, \mu)$ by $(1, A, i)$ in a way similar to what explained in $\S 4.2$. The sets of equivalence classes of singular and 2-fold special extensions of $(T, G, \mu)$ by $(1, A, i)$ become abelian groups under Baer sum.

Theorem 25. Let $\Phi=(T, G, \mu)$ be a crossed module, $A$ a $\pi_{1}$-module. Then $H^{2}(B(\Phi), A)$ is isomorphic to the group of equivalence classes of singular extensions of $(T, G, \mu)$ by $(1, A, i)$ and $H^{3}(B(\Phi), A)$ is isomorphic to the group of equivalence classes of 2-fold special extensions of $(T, G, \mu)$ by $(1, A, i)$.

Proof. From Proposition $21, H^{2}(B(\Phi), A) \cong H^{1} \operatorname{Der}\left(\mathbb{G}_{\bullet} \Phi,(1, A, i)\right)$ and $H^{3}(B(\Phi), A) \cong H^{2} \operatorname{Der}\left(\mathbb{G}_{\bullet} \Phi,(1, A, i)\right)$. By the interpretation in terms of extensions of the first and second cotriple cohomology groups in categories of interest given in [21, Theorems 2.1.3 and 2.2.3] the result follows.

\section{The relationship with cohomology of groups with opera- tors}

Our purpose in this section is to elucidate the relationship between the cohomology theory $D^{*}((T, G, \mu), A)$ of a crossed module $(T, G, \mu)$ with coefficients in a $\pi_{1}$-module $A$ and the cohomology $H_{G}^{*}(T, A)$ studied in [6]. The latter is the cohomology of a group $T$ endowed with a $G$-action by automorphisms with coefficients 
in a $G$-equivariant $T$-module $A$; this consists of an abelian group $A$ with actions of $T$ and $G$ such that

$$
{ }^{g}\left({ }^{t} a\right)={ }^{g} t\left({ }^{g} a\right), \quad g \in G, \quad t \in T \quad a \in A .
$$

The possibility that a relationship between the two theories may exist is suggested by the fact that, by $[\mathbf{6}, \mathrm{p} .11]$

$$
D^{0}((T, G, \mu), A) \cong \operatorname{Der}((T, G, \mu), A) \cong \operatorname{Hom}_{G}(T, A) \cong \operatorname{Der}_{G}(T, A) \cong H_{G}^{1}(T, A) .
$$

We shall also exhibit a counterexample showing that in general $D^{n}((T, G, \mu), A)$ and $H_{G}^{n+1}(T, A)$ are not isomorphic for $n>0$.

In order to establish the relationship with $H_{G}^{*}(T, A)$ we first prove that if $(T, G, \mu)$ is a precrossed module, $H_{G}^{n}(T, A)$ can be recovered as cohomology of a precrossed module for $n>0$.

Recall that a precrossed module $(T, G, \mu)$ consists of a group homomorphism $\mu: T \rightarrow G$ together with an action of $G$ on $T$ such that $\mu\left({ }^{g} t\right)=g \mu(t) g^{-1}, g \in G$, $t \in T$. A morphism of precrossed modules $(f, h):(T, G, \mu) \rightarrow\left(T^{\prime}, G^{\prime}, \mu^{\prime}\right)$ consists of group homomorphisms $f: T \rightarrow T^{\prime}, \quad h: G \rightarrow G^{\prime}$ with $f\left({ }^{g} t\right)=h(g) f(t), t \in T$, $g \in G$. Denote by PCM the category of precrossed modules. PCM is equivalent to the category of pre-cat ${ }^{1}$-groups. A pre-cat ${ }^{1}$-group is a group $G$ together with two endomorphisms $d_{0}, d_{1}: G \rightarrow G$ such that $d_{1} d_{0}=d_{0}, d_{0} d_{1}=d_{1}$. A morphism $f: G \rightarrow G^{\prime}$ of pre-cat ${ }^{1}$-groups is a group homomorphism commuting with $d_{0}, d_{1}$.

In $[\mathbf{1}]$ is proved that the category of precrossed modules is tripleable over Set; the corresponding cotriple is then used to define a cotriple (co)homology of precrossed modules with trivial coefficients. Following the same method used for crossed modules, we introduce a cotriple cohomology of precrossed modules with a system of local coefficients.

Notice that PCM is a category of interest in the sense of [17]; this follows from the fact that $\mathbf{P C M}$ is equivalent to pre-cat ${ }^{1}$-groups and from the tripleability of PCM over Set.

Lemma 26. Let $(T, G, \mu)$ be a precrossed module, $A$ an abelian group. Then $(A, 1,0)$ is a $(T, G, \mu)$-module (in the category of interest PCM) if and only if $A$ is a $G$ equivariant $T$-module in the sense of [6] and in this case

$$
\operatorname{Der}((T, G, \mu),(A, 1,0)) \cong \operatorname{Der}_{G}(T, A) .
$$

Proof. If $(A, 1,0)$ is a $(T, G, \mu)$-module, there is a split singular extension in PCM

$$
(A, 1,0) \longmapsto\left(T^{\prime}, G^{\prime}, \widetilde{\mu}\right) \rightleftarrows(T, G, \mu) .
$$

In particular there are split extensions of groups $A \longmapsto T^{\prime} \rightleftarrows T$ and $1 \longmapsto G^{\prime} \rightleftarrows G$, so that we can assume that $G^{\prime}=G$, and that $T^{\prime} \cong A \rtimes T$, where the action of $T$ on $A$ is by conjugation via the splitting; we can also assume that the map $A \rtimes T \rightarrow T$ is the projection and $T \rightarrow A \rtimes T$ is the inclusion. So we have the split extension in PCM

$$
(A, 1,0) \longrightarrow(A \rtimes T, G, \widetilde{\mu}) \underset{\left(i, \mathrm{id}_{G}\right)}{\stackrel{\left(\mathrm{pr}_{T}, \mathrm{id}_{G}\right)}{\rightleftarrows}}(T, G, \mu) .
$$


The action of $G$ on $A \rtimes T$ induces an action of $G$ on $A$; in fact, since $\left(\operatorname{pr}_{T}, \operatorname{id}_{G}\right)$ is a map of precrossed modules, $\operatorname{pr}_{T}\left({ }^{g}(a, 1)\right)=1$ for all $g \in G, a \in A$. Since the maps in the split extension (38) are maps of precrossed modules, we have, for all $a \in A, \quad g \in G, \quad t \in T, \widetilde{\mu}(a, t)=\mu(t),{ }^{g}(a, t)={ }^{g}(a, 1){ }^{g}(0, t)=\left({ }^{g} a, 1\right)\left(0,{ }^{g} t\right)=$ $\left({ }^{g} a,{ }^{g} t\right)$. In particular we obtain, for all $a \in A, \quad g \in G, \quad t \in T,\left({ }^{g}\left({ }^{t} a\right),{ }^{g} t\right)=$ ${ }^{g}\left({ }^{t} a, t\right)={ }^{g}((0, t)(a, 1))=\left(0,{ }^{g} t\right)\left({ }^{g} a, 1\right)=\left({ }^{g} t\left({ }^{g} a\right),{ }^{g} t\right)$. Hence ${ }^{g}\left({ }^{t} a\right)={ }^{g} t\left({ }^{g} a\right)$ so that, in the terminology of [6] $A$ is a $G$-equivariant $T$-module. Conversely if $A$ is a $G$-equivariant $T$-module, $(38)$ is a split singular extension in PCM, so $(A, 1,0)$ is a $(T, G, \mu)$-module. We have

$$
\operatorname{Der}((T, G, \mu),(A, 1,0)) \cong \operatorname{Hom}_{\mathbf{P C M} /(T, G, \mu)}((T, G, \mu),(A \rtimes T, G, \widetilde{\mu})) .
$$

We now show that there is an isomorphism

$$
\alpha: \quad \operatorname{Hom}_{\mathbf{P C M} /(T, G, \mu)}((T, G, \mu),(A \rtimes T, G, \widetilde{\mu})) \rightarrow \operatorname{Der}_{G}(T, A) .
$$

Let $\alpha\left(\left(D, \operatorname{id}_{T}\right), \operatorname{id}_{G}\right)=D$. Notice that $D \in \operatorname{Der}_{G}(T, A)$; in fact $D \in \operatorname{Der}(T, A)$ and since $\left(\left(D, \mathrm{id}_{T}\right), \mathrm{id}_{G}\right)$ is a morphism of precrossed modules, $\left(D\left({ }^{g} t\right),{ }^{g} t\right)={ }^{g}(D(t), t)$ so that $D\left({ }^{g} t\right)={ }^{g} D(t)$. Clearly $\alpha$ is injective. Let $D \in \operatorname{Der}_{G}(T, A)$. Then $\left(\left(D, \mathrm{id}_{T}\right), \mathrm{id}_{G}\right)$ is a morphism of precrossed modules over $(T, G, \mu)$ and $\alpha\left(\left(D, \mathrm{id}_{T}\right), \mathrm{id}_{G}\right)=D$

Notice that if $(T, G, \mu)$ is a crossed module and $A$ is a $\pi_{1}$-module, $A$ is a $G$ equivariant trivial $T$-module in the terminology of $[\mathbf{6}$, p. 15]. If $I: \mathbf{C M} \rightarrow \mathbf{P C M}$ is the inclusion, we have in this case

$$
\operatorname{Der}(I(T, G, \mu),(A, 1,0)) \cong \operatorname{Der}((T, G, \mu), A) .
$$

\section{Remark 27.}

An equivalent version of Lemma 26 is obtained by working in pre-cat ${ }^{1}$-groups rather than in PCM. Let $(A, 0,0)$ and $\left(T \rtimes G, d_{0}, d_{1}\right)$ be the pre-cat ${ }^{1}$-groups corresponding to the precrossed modules $(A, 1,0)$ and $(T, G, \mu)$ respectively. It is easily checked that $(A, 0,0)$ is a $\left(T \rtimes G, d_{0}, d_{1}\right)$-module if and only if $A$ is a $(T \rtimes G)$-module and

$$
\operatorname{Der}\left(\left(T \rtimes G, d_{0}, d_{1}\right),(A, 0,0)\right) \cong\{D \in \operatorname{Der}(T \rtimes G, A) \mid D(1, G)=0\} .
$$

The two versions of the lemma are clearly equivalent. Recall in fact [6, Theorem $2.2]$ that the categories of $G$-equivariant $T$-modules and that of $(T \rtimes G)$-modules are equivalent, with the action of $T \rtimes G$ on a $G$-equivariant $T$-module $A$ given by ${ }^{(t, g)} a={ }^{t}\left({ }^{g} a\right)$; moreover it is straightforward to check that there is an isomorphism

$$
\alpha: \operatorname{Der}_{G}(T, A) \rightarrow\{D \in \operatorname{Der}(T \rtimes G, A) \mid D(1, G)=0\}
$$

given by $\alpha(D)(t, g)=D(t),(t, g) \in T \rtimes G$.

Let $\overline{\mathbb{G}}$ be the cotriple on PCM of $[\mathbf{1}]$.

Proposition 28. Let $(T, G, \mu)$ be a precrossed module, $A$ a $G$-equivariant T-module. Then for each $n \geqslant 0$

$$
H^{n} \operatorname{Der}\left(\overline{\mathbb{G}}_{\bullet}(T, G, \mu),(A, 1,0)\right) \cong H_{G}^{n+1}(T, A) .
$$


Proof. From [6, Theorem 2.6] for each $n \geqslant 0$

$$
H_{G}^{n}(T, A) \cong H^{n}\left(B_{T \rtimes G}, B_{G}, A\right) \cong H^{n}\left(\operatorname{ker}\left(C^{*}(T \rtimes G, A) \stackrel{r}{\rightarrow} C^{*}(G, A)\right),\right.
$$

where $C^{*}(T \rtimes G, A)$ and $C^{*}(G, A)$ are the ordinary cochain complexes for computing group cohomology and $r_{\bullet}$ are the restriction maps.

Denote $\overline{\mathbb{G}}_{\bullet}(T, G, \mu)=\left(T_{\bullet}, G_{\bullet}, \mu_{\bullet}\right)$. From Lemma 26 and Remark 27, there is a short exact sequence for each $n$

$$
0 \rightarrow \operatorname{Der}\left(\left(T_{n}, G_{n}, \mu_{n}\right),(A, 1,0)\right) \rightarrow \operatorname{Der}\left(T_{n} \rtimes G_{n}, A\right) \rightarrow \operatorname{Der}\left(G_{n}, A\right) \rightarrow 0
$$

where the map $\operatorname{Der}\left(T_{n} \rtimes G_{n}, A\right) \rightarrow \operatorname{Der}\left(G_{n}, A\right)$ is restriction. It is proved in [1, p. $12]$ that $T_{\bullet} \rtimes G_{\bullet} \rightarrow T \rtimes G$ and $G_{\bullet} \rightarrow G$ are free simplicial resolutions. Hence, if $\perp$ is the ordinary free cotriple on Groups we obtain short exact sequences of cochain complexes

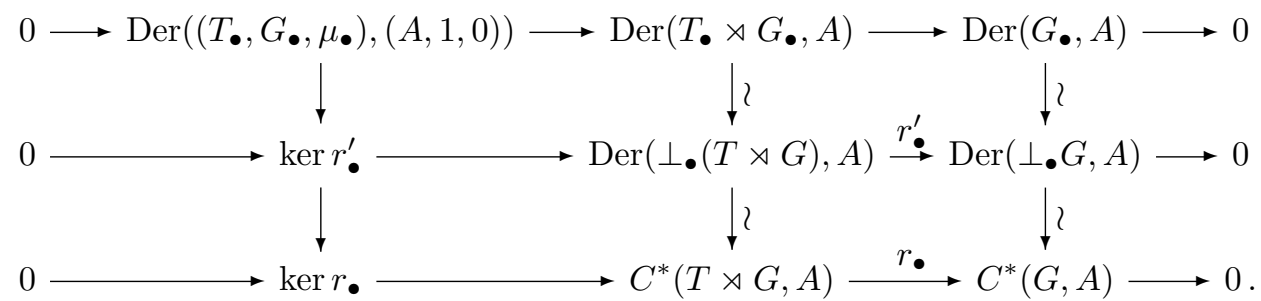

In the above diagram the maps $\operatorname{Der}\left(T_{\bullet} \rtimes G_{\bullet}, A\right) \stackrel{\sim}{\rightarrow} \operatorname{Der}\left(\perp_{\bullet}(T \rtimes G), A\right), \operatorname{Der}\left(G_{\bullet}, A\right) \stackrel{\sim}{\rightarrow}$ $\operatorname{Der}\left(\perp_{\bullet} G, A\right)$ are homotopy equivalences, and $\operatorname{Der}\left(\perp_{\bullet}(T \rtimes G), A\right) \rightarrow C^{*}(T \rtimes G, A)$, $\operatorname{Der}(\perp \cdot G, A) \rightarrow C^{*}(G, A)$ are the natural cochain maps of the Barr-Beck theory which induce isomorphisms in cohomology (see [2]). Taking the long exact cohomology sequences in each row of the above diagram and applying the Five Lemma we obtain for all $n>0$

$$
H^{n} \operatorname{Der}\left(\left(T_{\bullet}, G_{\bullet}, \mu_{\bullet}\right),(A, 1,0)\right) \cong H^{n} \operatorname{ker} r_{\bullet}^{\prime} \cong H^{n+1} \operatorname{ker} r_{\bullet} \cong H_{G}^{n+1}(T, A) .
$$

Finally, by Lemma 26 , by [6, p. 11] and by general properties of cotriple cohomology $H^{0} \operatorname{Der}\left(\left(T_{\bullet}, G_{\bullet}, \mu_{\bullet}\right),(A, 1,0)\right) \cong \operatorname{Der}((T, G, \mu),(A, 1,0)) \cong \operatorname{Der}_{G}(T, A) \cong H_{G}^{1}(T, A)$.

Theorem 29. Let $(T, G, \mu)$ be a crossed module, $A$ a $\pi_{1}$-module. Let $s_{n}: \overline{\mathbb{G}}^{n} I \Rightarrow$ $I \mathbb{G}^{n}, I: \mathbf{C M} \hookrightarrow \mathbf{P C M}$ be as in $[\mathbf{1}]$ and $\bar{s}_{n}=\operatorname{Der}\left(s_{n}, A\right)$. There exists a long exact cohomology sequence

$$
\begin{aligned}
0 & \rightarrow H^{0} \operatorname{coker} \bar{s}_{\bullet} \rightarrow D^{1}((T, G, \mu), A) \rightarrow H_{G}^{2}(T, A) \rightarrow H^{1} \operatorname{coker} \bar{s}_{\bullet} \rightarrow \\
& \rightarrow D^{2}((T, G, \mu), A) \rightarrow H_{G}^{3}(T, A) \rightarrow \cdots
\end{aligned}
$$

Proof. It is proved in $[\mathbf{1}$, p. 14] that there exists a surjective homomorphism of resolutions

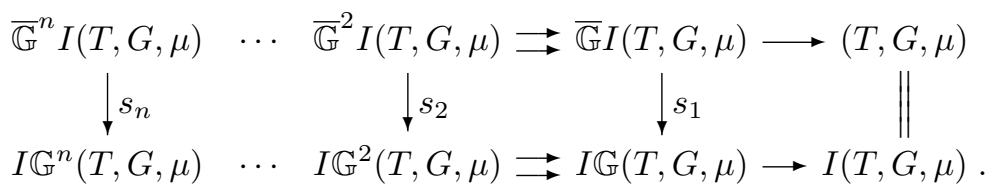


The natural transformation $s_{n}: \overline{\mathbb{G}}^{n} I \rightarrow I \mathbb{G}^{n}$ is defined inductively by $s_{n+1}=$ $s_{n} \mathbb{G} \circ \overline{\mathbb{G}}^{n} s_{1}$ for every $n \geqslant 1$, and $s_{1}$ is the natural transformation sending each crossed module $(T, G, \mu)$ to the canonical projection $\overline{\mathbb{G}}(T, G, \mu) \rightarrow \mathcal{P} \overline{\mathbb{G}}(T, G, \mu)=$ $\mathbb{G}(T, G, \mu)$ where $\mathcal{P}: \mathbf{P C M} \rightarrow \mathbf{C M}$ is Peiffer abelianization [1]. Hence (39) gives rise to an injective morphism of cochain complexes

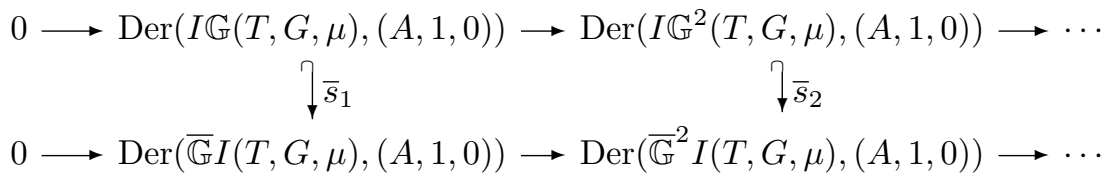

Since $\operatorname{Der}\left(I \mathbb{G}_{\bullet}(T, G, \mu),(A, 1,0)\right) \cong \operatorname{Der}\left(\mathbb{G}_{\bullet}(T, G, \mu), A\right)$ we therefore have a short exact sequence of cochain complexes

$$
\operatorname{Der}\left(\mathbb{G}_{\bullet}(T, G, \mu), A\right) \stackrel{\bar{s}_{\bullet}}{\mapsto} \operatorname{Der}\left(\overline{\mathbb{G}}_{\bullet} I(T, G, \mu),(A, 1,0)\right) \rightarrow \operatorname{coker} \bar{s}_{\bullet} .
$$

Since $H^{0} \operatorname{Der}\left(\mathbb{G}_{\bullet}(T, G, \mu), A\right) \cong H^{0} \operatorname{Der}\left(\overline{\mathbb{G}}_{\bullet} I(T, G, \mu), A\right) \cong \operatorname{Der}_{G}(T, A)$, taking the corresponding long exact cohomology sequence and using Proposition 28 the result follows.

The following counterexample shows that in general $D^{n}((T, G, \mu), A)$ and $H_{G}^{n+1}(T, A)$ are not isomorphic for $n>0$. Let $C_{\infty}$ be the infinite cyclic group with generator $t$, let $H$ be the subgroup of $C_{\infty}$ generated by $t^{2}$ so that $C_{\infty} / H \cong C_{2}$, the cyclic group of order 2. Consider the crossed module $\Phi=\left(H, C_{\infty}, i\right)$ and let $A$ be a $C_{2}$-module. Let $\sigma$ be the generator of $C_{2}$ and $N=1+\sigma$; since $H^{n}\left(C_{\infty}, A\right)=0$ for $n>1$ by Theorem 7

$$
D^{n}(\Phi, A) \cong H^{n+1}\left(C_{2}, C_{\infty} ; A\right) \cong H^{n+2}\left(C_{2} ; A\right) \cong\left\{\begin{array}{l}
\{a \in A: N a=0\} /(\sigma-1) A \quad n \text { odd, } \\
A^{C_{2} / N A} \quad n \text { even, } n>0 .
\end{array}\right.
$$

On the other hand, since $H$ acts trivially on $A$, in the terminology of $[6] A$ is a $C_{\infty}$-equivariant trivial $H$-module, so that by [6, Corollary 3.7] for all $n \geqslant 1$

$$
H_{C_{\infty}}^{n+1}(H, A) \cong H^{n+1}\left(H \rtimes C_{\infty}, A\right) .
$$

Since the action of $C_{\infty}$ on $H$ is trivial, $H \rtimes C_{\infty} \cong H \times C_{\infty}$. From the Lyndon / Hochschild - Serre spectral sequence

$$
E_{2}^{p q}=H^{p}\left(C_{\infty}, H^{q}(H, A)\right) \Rightarrow H^{p+q}\left(H \times C_{\infty}, A\right),
$$

since $E_{2}^{p q}=0$ for $p \neq 0,1$, there are exact sequences, for each $n \geqslant 1$

$$
H^{1}\left(C_{\infty}, H^{n-1}(H, A)\right) \longmapsto H^{n}\left(H \times C_{\infty}, A\right) \rightarrow H^{0}\left(C_{\infty}, H^{n}(H, A)\right) .
$$

Since $H^{n}(H, A)=0$ for $n \geqslant 2, H^{1}(H, A) \cong A$, we obtain

$$
H_{C_{\infty}}^{n+1}(H, A) \cong \begin{cases}H^{1}\left(C_{\infty}, A\right) \cong A_{C_{\infty}} & n=1 \\ 0 & n>1 .\end{cases}
$$

Hence in general $D^{n}(\Phi, A) \neq H_{C_{\infty}}^{n+1}(H, A)$ for $n>0$.

We finally remark that, since $H_{i}(H)=0$ for all $i \geqslant 2$, from [6, p. 19] there are isomorphisms $H_{C_{\infty}}^{n+1}(H, A) \cong \operatorname{Ext}_{C_{\infty}}^{n}\left(H_{a b}, A\right)$ for all $n \geqslant 0$. Hence this counterexample also shows that despite the isomorphism $D^{0}((T, G, \mu), A) \cong \operatorname{Hom}_{G}(T, A) \cong$ 
$\operatorname{Hom}_{G \text {-Mod }}\left(T_{a b}, A\right)$ for any crossed module $(T, G, \mu)$, in general the cohomology groups $D^{n}((T, G, \mu), A)$ and $H_{G \text {-Mod }}^{n}\left(T_{a b}, A\right)$ are not isomorphic for $n>0$.

\section{References}

[1] D. Arias, M. Ladra, A. R. Grandjean, Homology of precrossed modules, Illinois Journal of Math., 46, (2002) no. 3, 739-754.

[2] M. Barr and J. Beck, Acyclic models and triples, in Proceedings of the Conference on Categorical Algebra (La Jolla), Springer, (1966), 336-343.

[3] M. Barr and J. Beck, Homology and standard constructions, in Seminar on triples and categorical homology theory, Lect. Notes in Math, vol. 80, Springer (1969), 245-335.

[4] P. Carrasco, A. N. Cegarra, A. R. Grandjean, (Co)-homology of crossed modules, Journal of Pure and Applied Algebra 168 (2002), 147-176.

[5] H. Cartan, S. Eilenberg, Homological Algebra, Princeton University Press, 1956.

[6] A. M. Cegarra, J. M. Garcia - Calcines and J. A. Ortega, Cohomology of groups with operators, Homology, Homotopy and Application, vol. 4 (1), (2002), 1-23.

[7] T. Datuashvili, Cohomologically trivial internal categories in categories of groups with operations. Applied Categorical Structures 3, n. 3, (1995), 221-237.

[8] T. Datuashvili, T. Pirashvili, On (co)homology of 2-types and crossed modules, Journal of Algebra 244, (2001), 352-365.

[9] J. W. Duskin, Simplicial methods and interpretation of "triple" cohomology, Mem. A.M.S., vol. 3, issue 2, n. 163, (1975).

[10] G. J. Ellis, Crossed modules and their higher dimensional analogues, Thesis, University of Bangor, (1984).

[11] G. J. Ellis, Homology of 2-types. J. London Math. Soc. (2) 46, n. 1, (1992), $1-27$.

[12] P. G. Goerss, J. F. Jardine, Simplicial Homotopy Theory, Birkäuser, (1991).

[13] A. R. Grandjean, M. Ladra, T. Pirashvili, CCG-homology of crossed modules via classifying spaces. Journal of Algebra 229, n. 2, (2000), 660-665.

[14] J. L. Loday, Cohomologie et groupe de Steinberg relatifs, Journal of Algebra 54, n. 1, (1978), 178-202.

[15] J. L. Loday, Spaces with finitely many non-trivial homotopy groups, Journal of Pure and Appl. Algebra 24, n. 2, (1982), 179-202.

[16] K. Norrie, Actions and automorphisms of crossed modules, Bull. Soc. Math. France, 118, (1990), 129-146.

[17] G. Orzech, Obstruction theory in algebraic categories I and II, J. Pure and Applied Algebra 2, (1972), 287-314 and 315-340. 
[18] S. Paoli, Developments in the (co)homology of crossed modules, PhD thesis, University of Warwick, November 2002.

[19] T. Porter, Extensions, crossed modules and internal categories in categories of groups with operations. Proc. Edimburgh Math. Soc. 30, n. 3, (1987), 373-381.

[20] D. Quillen, Higher algebraic K-theory, Lecture notes in Mathematics, vol. 341, Springer-Verlag, (1973).

[21] M. J. Vale, Torsors and special extensions, Cahiers de Topologie et Géométrie Différentielle Catégorique, 26, n. 1, (1985), 63-90.

[22] A. M. Vieites Rodriguez, J. M. Casas Miras, Derivations of crossed modules, Extracta Mathematicae 14, n. 1, (1999), 63-67.

This article may be accessed via WWW at http://www.rmi.acnet.ge/hha/ or by anonymous ftp at

ftp://ftp.rmi.acnet.ge/pub/hha/volumes/2003/n1a12/v5n1a12.(dvi,ps,pdf)

Simona Paoli simona@maths. warwick.ac.uk

Mathematics Institute

University of Warwick

Coventry CV4 7AL

England 